\title{
Mechanism for remodelling of the cell cycle checkpoint protein Mad2 by the ATPase TRIP13
}

\author{
Claudio Alfieri $^{1}$, Leifu Chang ${ }^{1}$, and David Barford ${ }^{1}$ \\ ${ }^{1} \mathrm{MRC}$ Laboratory of Molecular Biology, Francis Crick Avenue, Cambridge, CB2 0QH, UK
}

\section{Abstract}

The maintenance of genome stability in mitosis is coordinated by the spindle assembly checkpoint (SAC) through its effector the mitotic checkpoint complex (MCC), an APC/C inhibitor 1,2. Unattached kinetochores control MCC assembly by catalysing a change in topology of the $\beta$-sheet of Mad2, an MCC subunit, generating the active closed Mad2 conformer (C-Mad2) 3-5.

Disassembly of free MCC, required for SAC inactivation and chromosome segregation, is an ATPdependent process driven by the AAA+ ATPase TRIP13. In combination with the SAC antagonist p31 ${ }^{\text {comet }}$ (ref. 6), TRIP13 remodels C-Mad2 into inactive open Mad2 (O-Mad2) 7-10. Here, we present a mechanism to explain how TRIP13-p31 ${ }^{\text {comet }}$ disassembles the MCC. Cryo-EM structures of the TRIP13-p31 ${ }^{\text {comet: }} \mathrm{C}-\mathrm{Mad} 2-\mathrm{Cdc} 20$ complex reveal that $\mathrm{p} 31^{\text {comet }}$ recruits C-Mad2 to a defined site on the TRIP13 hexameric ring, positioning the C-Mad2 N-terminus (Mad2 ${ }^{\mathrm{NT}}$ ) to insert into the axial pore of TRIP13, and distorting the TRIP13 ring to initiate remodelling. Molecular modelling suggests that by gripping Mad2 ${ }^{\mathrm{NT}}$ within its axial pore, TRIP13 couples sequential ATP-driven translocation of its hexameric ring along Mad2 ${ }^{\mathrm{NT}}$ to push upwards on, and simultaneously rotate, the globular domains of the $\mathrm{p} 31^{\text {comet:}} \mathrm{C}-\mathrm{Mad} 2$ complex. This unwinds a region of the aA helix of C-Mad2 required for stabilizing the C-Mad2 $\beta$-sheet, thus destabilizing C-Mad2 in favour of O-Mad2 and dissociating Mad2 from $\mathrm{p} 31^{\text {comet }}$. Our study provides insights into how specific substrates are recruited to AAA+ ATPases through adaptor proteins and suggests a model of how translocation through the axial pore of AAA+ ATPases is coupled to protein remodelling.

To investigate MCC disassembly catalysed by the joint action of $\mathrm{p} 31^{\text {comet }}$ and TRIP13, the two proteins were incubated with ATP and either free MCC or MCC in complex with the $\mathrm{APC} / \mathrm{C}\left(\mathrm{APC} / \mathrm{C}^{\mathrm{MCC}}\right)$. $\mathrm{p} 31^{\text {comet }}$ and TRIP13 catalysed formation of O-Mad2 from the MCC much more effectively than from $\mathrm{APC} / \mathrm{C}^{\mathrm{MCC}}$ (Extended Data Fig. 1a, lanes 5-6, 7-8 and

Correspondence and request for material should be addressed to D.B. (dbarford@mrc-lmb.cam.ac.uk).

Reporting summary. Further information on experimental design is available in the Nature Research Reporting Summary linked to this paper.

Data availability. Figure source data are provided in Supplementary Fig. 1. Cryo-EM maps of the TRIP13-p31 ${ }^{\text {comet }}$-substrate complex have been deposited with the Electron Microscopy Data Bank under accession numbers EMD-4166. Atomic coordinates of the TRIP13-p31 ${ }^{\text {comet }}$-substrate complex have been deposited with RCSB under entry ID 6F0X.

Author contributions. C.A. cloned bacterially expressed $\mathrm{p} 31^{\text {comet }}$, Mad2 and TRIP13 wild type and mutant constructs, purified proteins, performed the protein complex reconstitutions and biochemical analysis and mutagenesis. C.A. prepared EM grids, analysed electron microscopy data and determined the three dimensional reconstructions. C.A. collected electron microscopy data with the help of L.C. C.A. fitted coordinates, built models. D.B. directed the project. C.A. and D.B. wrote the manuscript with input from L.C.

Author information. The authors declare no competing interests. 
Extended Data Figs 1b and 2a). Release of O-Mad2 from APC/C $\mathrm{C}^{\mathrm{MCC}}$ does not require the APC/C subunit Apc15 (Extended Data Fig. 1a, lanes 1-2 and 5-6), consistent with TRIP13$\mathrm{p} 31^{\text {comet }}$ and the APC/C mediating independent SAC silencing pathways 8,9,11-16. The TRIP13 catalytic mutant (TRIP13 ${ }^{\mathrm{E} 253 \mathrm{Q}}$ ) 17 is defective in generating O-Mad2 (Extended Data Fig. 1a, lanes 3-4, 9-10 and Extended Data Fig. 1b). On incubation with the MCC and p31 ${ }^{\text {comet }}$, a TRIP13 ${ }^{\mathrm{E} 253 \mathrm{Q}}$ hexamer formed a complex with p31 ${ }^{\text {comet }}, \mathrm{Cdc} 20$ and Mad2 in a 1:1:1:1 stoichiometry, agreeing with 17,18 (Extended Data Fig. 1c). p31 ${ }^{\text {comet }}$ but not TRIP13 interacts with the free MCC and APC/CMCC (Extended Data Fig. 1b,d). The

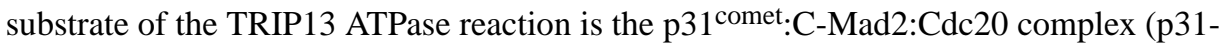
substrate) which forms a tight complex with TRIP13 ${ }^{\mathrm{E} 253 \mathrm{Q}}$ (Extended Data Fig. 1e).

We then determined the cryo-EM structure of the TRIP13 ${ }^{\mathrm{E} 253 \mathrm{Q}}$-p31-substrate complex with ATP $\gamma \mathrm{S}$ (Extended data Fig. 1e). Refinement of the EM dataset yielded a 3D reconstruction at $4.5 \AA$ resolution, extending to $4.3 \AA$ for focussed refinement of TRIP13 monomers A-D (Extended Data Fig. 3 and Extended Data Table 1). 3D classification revealed three structural classes (Extended Data Fig. 4). Class 1 is the unliganded apo TRIP13 hexamer with classes 2 and 3 belonging to two distinct conformational states of the TRIP13 ${ }^{\mathrm{E} 253 \mathrm{Q}}$ p31-substrate complex. TRIP13 in class 2 represents the basal state whereas class 3 presents structural rearrangements in TRIP13, suggestive of an activated state. An atomic model for apo TRIP13 was built into the overall EM density map, guided by the crystal structure of a TRIP13 monomer 18 (Fig. 1a and Extended Data Fig. 3c,d and Extended Data Table 1). In the cryo-EM structure, TRIP13 forms a closed hexameric ring featuring a central pore, whereas in the crystal structure adjacent subunits assemble into a helical filament 18 (Fig. 1a, Extended Data Fig. 5a). The TRIP13 cryo-EM structure also differs from the flat closed ring of the C. elegans TRIP13 ortholog PCH2 17 (Fig. 1a and Extended Data Fig. 5a).

Our cryo-EM structure shows that with ATP $\gamma \mathrm{S}$ the TRIP13 ring adopts a compact conformation with a convex top surface and concave lower surface (Fig. 1a and Extended Data Fig. 5a). Monomers A to E form a right-handed spiral. Monomer F, structurally distinct from its counterparts due to the separation of its large and small AAA+ domains, although less pronounced than that of the open subunits of PCH2 (ref. 17) (Extended Data Fig. 5b), forms a seam in the hexameric ring bridging monomers A and E (Fig. 1a and Extended Data Fig. 5a,b). Clear EM density is visible for ATP $\gamma \mathrm{S}$ at monomers A to E (Extended Data Fig. 3e). Pore loops- 1 that in the $\mathrm{PCH} 2$ flat ring conformation occlude the central pore, undergo a rotation upwards (Extended Data Fig. 5c) such that Trp221 and Phe222 of monomer A are exposed on the surface.

To build the basal state TRIP13 ${ }^{\mathrm{E} 253 \mathrm{Q}}$-p31-substrate model (class 2 of the 3D classification,

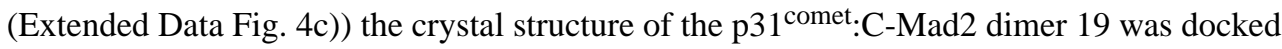
into the large EM density feature above the convex face of TRIP13 (Fig. $1 \mathrm{~b}$ and Extended

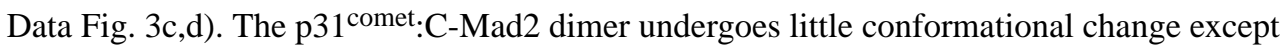
for a structural rearrangement of Mad2 ${ }^{\mathrm{NT}}$ (Extended Data Fig. 6a,b). The conformation of the TRIP13 ring is essentially identical to apo TRIP13 (Extended Data Fig. 4b). The main contact between the $\mathrm{p} 31$-substrate and TRIP13 involves a conserved basic surface on p3 $1^{\text {comet }}$ opposite the C-Mad2 interface (Figs 1b and 2a,b and Supplementary Video 1). Strikingly, $\mathrm{p} 31^{\text {comet }}$ positions C-Mad2 so that $\mathrm{Mad}^{\mathrm{NT}}$, extending from the start of the $\mathrm{aA}$ 
helix, is precisely located to enter the TRIP13 pore (Figs 1b and 3a and Extended Data Fig. 3d,f). Notably, structural elements of C-Mad2 that are remodelled on conversion to O-Mad2 - the $\beta 8$ '- $\beta 8$ " hairpin and safety belt (Extended Data Fig. $6 \mathrm{~b}, \mathrm{c}$ ) - form no direct contacts to TRIP13.

TRIP13 interacts with $\mathrm{p} 31^{\text {comet }}$ through a conserved and negatively charged surface on monomers D and E. Monomer D interacts with the safety belt of $\mathrm{p} 31^{\text {comet }}$, with monomer $\mathrm{E}$ contacting conserved basic residues of the extended p31 ${ }^{\text {comet }} a 3-4$ loop (Figs $1 b$ and $2 b$ and Extended Data Fig. 7a-c), consistent with 17,18,20. Combined deletion of seven basic residues of the a 3-4 loop severely ablated TRIP13-catalysed Mad2 remodelling, without disrupting complex assembly (Fig. 2c and Extended Data Figs 2c and 7d).

In the TRIP13-substrate complex, C-Mad2 interacts tightly with p31 ${ }^{\text {comet }}$ through the Mad2 dimerization interface 19,21-23 and entraps the Mad2-binding motif (MBM) of Cdc20 under its safety belt (Fig. 1b). No other density for Cdc20 was visible (Extended Data Fig. $3 \mathrm{c}, \mathrm{d})$. In agreement with 18, the 109-117 loop of C-Mad2 interacts with pore loop-1 of TRIP monomer A. Mad2 $2^{\mathrm{NT}}$ adopts a fully extended conformation with residues 2-9 inserting midway into the narrow pore at the centre of TRIP13 (Figs 1b and 3a and Extended Data Fig. 3d,f and Supplementary Video 1). In this configuration, the conserved TRIP13 pore loop-1 Trp221 and Phe222 of monomers B to D embrace residues 2 to $7 \mathrm{Mad} 2^{\mathrm{NT}}$ (Fig. 3a and Extended Data Fig. 3f). Trp221 and Phe222 are responsible for Mad2 processing 17. Pore loop-1 of monomer E is too low in the TRIP13 spiral to contact Mad2 ${ }^{\mathrm{NT}}$ (Fig. 3a), whereas that of monomer $\mathrm{F}$ is disordered.

Monomer A of TRIP13 also interacts with Mad2 ${ }^{\mathrm{NT}}$ through the underlying acidic pore loop-2 (Fig. 3a), contributing a key specificity determinant for Mad2 ${ }^{\mathrm{NT}}$. The region of $\mathrm{Mad} 2{ }^{\mathrm{NT}}$ that forms intimate contacts to conserved pore loop residues of monomers A to D is hydrophobic and basic $\left(\mathrm{L}^{3} \mathrm{QLSR}{ }^{7}\right)$ (Fig. 3a). Although EM density for side chains of $\mathrm{Mad} 2{ }^{\mathrm{NT}}$ and pore loops-2 is incomplete, it is indicative of interactions between $\mathrm{Arg} 7$ of Mad2 ${ }^{\mathrm{NT}}$ and Glu269 and Asp272 of pore loop-2 of monomer A, with Leu3 and Leu5 of $\operatorname{Mad} 2^{\mathrm{NT}}$ contacting the Phe222 side chains of pore loops- 1 of monomers $\mathrm{C}$ and $\mathrm{B}$, respectively.

Importantly, the Mad2 ${ }^{\mathrm{NT}} \Phi^{3} \mathrm{QLSR}^{7}$ motif is conserved in Mad2 homologues and other TRIP13 HORMA-domain proteins substrates (ref. 18) (Extended Data Fig. 8c). To test the importance of the Mad2 ${ }^{\mathrm{NT}}$ LSR sequence, we compared the efficacy of O-Mad2 release from a C-Mad2:Cdc20 complex incorporating either wild type C-Mad2 or a mutant with LSR replaced by LEE. Release of O-Mad2 was reduced in the LEE mutant (Fig. 3b, lanes 3-4, 7-8 and Extended Data Fig. 2c), showing that a positively charged segment in Mad2 ${ }^{\mathrm{NT}}$ is important for TRIP13 function. A TRIP13 mutant with either Ala or Arg substitutions of Glu269 and Asp272 was completely defective in Mad2 remodelling (Fig. 3c and Extended Data Fig. 2c). Removal of the first seven residues of Mad2 severely impaired O-Mad2 release (Fig. 2b, lanes 3-4, 5-6 and Extended Data Fig. 2b), however, deleting up to nine residues from the Mad2 N-terminus did not affect complex assembly (Extended Data Fig. 7d). These findings agree with a previous study that deletion of the first five residues of $\operatorname{Mad} 2(\Delta \mathrm{N} 5-\mathrm{Mad} 2)$ abolished both TRIP13's ability to convert C-Mad2 to O-Mad2, and the 
substrate-dependent stimulation of TRIP13 ATPase activity 18. In cells, whereas wild type Mad2 is in the open state 18,20, the $\Delta$ N5-Mad2 mutant exists $50 \%$ in the closed state and impairs SAC silencing 18. Deleting the first ten residues resulted in no cellular O-Mad2, and caused a more severe mitotic defect 18 .

AAA+ ATPases are molecular motors that convert the chemical energy of ATP hydrolysis into mechanical energy 24. To explore how TRIP13 ATPase activity induces conformational changes in C-Mad2 we modelled the likely structure of the TRIP13:p31-substrate complex after the first cycle of catalysis (Fig. 4b and Extended Data Fig. 9e and Supplementary Video2). The largest structural change involves the seam monomer $\mathrm{F}$ and neighbouring monomer E. Monomer F adopts an active (ATP-bound) conformation, translates $\sim 12 \AA$ to the top of the AAA+ spiral and establishes contacts with $\mathrm{Mad}_{2}{ }^{\mathrm{NT}}$ two residues along relative to monomer A (Fig. 4a,b and Extended Data Fig. 9b). Thus monomers $\mathrm{E}$ and $\mathrm{F}$ ( $\mathrm{E}^{1}$ and $\mathrm{F}^{1}$ in Fig. $4 \mathrm{~b}$ and Extended Data Fig. 9b) (superscript ' 1 ' denotes post catalytic cycle 1) climb relative to $\mathrm{C}-\mathrm{Mad} 2$, a process that pushes monomer $\mathrm{F}^{1}$ onto the $\mathrm{aA}$ and $\mathrm{aC}$ helices of $\mathrm{C}$ Mad2 (Fig. 4b and Extended Data Fig. 9b,c). The new conformation of monomer $\mathrm{E}^{\left(\mathrm{E}^{1} \text { in }\right.}$ Fig. 4b), not only differs, thereby disrupting the TRIP13:p31 ${ }^{\text {comet }}$ interface, but also severely clashes with p31 ${ }^{\text {comet }}$ (Extended Data Fig. 9c and Supplementary Video3). Due to these steric clashes, the globular portion of the p31-substrate moves vertically relative to Mad2 ${ }^{\mathrm{NT}}$ held within the TRIP13 pore by subunits $\mathrm{A}^{1}$ to $\mathrm{D}^{1}$ (Extended Data Fig. 9a,d). An anticlockwise rotation of the globular portion of the p31-substrate allows $\mathrm{p} 31^{\text {comet }}$ to engage the reset TRIP13 interface $60^{\circ}$ anti-clockwise. Were the entire p31-substrate (including $\mathrm{Mad} 2{ }^{\mathrm{NT}}$ ) to shift, the Leu13 Ca atom (the start of aA of C-Mad2) would be displaced $7.6 \AA$ after the first catalytic step (Extended Data Fig. 9i). However, because Mad2 ${ }^{\mathrm{NT}}$ is gripped by the TRIP13 pore, fixing Leu13 $\mathrm{Ca}$, the aA helix unwinds to stretch the polypeptide chain (Extended Data Fig. 9f-j). Modelling indicates that each round of catalysis causes a single a-helical turn to unwind into an extended conformation, coupled with $\mathrm{Mad}_{2}{ }^{\mathrm{NT}}$ progressing through the TRIP13 pore by two residues (Fig. 4 and Extended Data Fig. 9g-j and Supplementary Video2).

Unwinding the $a \mathrm{~A}$ helix suggests that the C-Mad2 to O-Mad2 conversion occurs through disrupting contacts with the adjacent $\beta 8$ '- $\beta$ 8" hairpin, including hydrogen bonds linking Ser16 of aA with Thr188 and His191 of $\beta 8$ '- $\beta 8$ "' (Fig. 4g,h and Supplementary Video 2). Since these hydrogen bonds stabilize C-Mad2 (refs 18,25), their loss would displace $\beta 8$ '$\beta 8$ '. Opening the buckle ( $\beta 8^{\prime}-\beta 8^{\prime}$ ' pairing with $\beta 5$ ), releases the safety belt structure that traps Cdc20 to C-Mad2, liberating Cdc20 (Extended Data Fig. 6d). Restructuring $\beta 8$ '- $\beta 8$ "' and the C-Mad2 C-terminus weakens interactions with $\mathrm{p} 31^{\text {comet }}$ that binds C-Mad2 (refs 19,21,22) (Extended Data Fig. 6e). As proposed 17, this coordinates remodelling of C-Mad2 with release from TRIP13, preventing substantial unfolding of Mad2 (ref. 17). Release of $\operatorname{Mad} 2^{\mathrm{NT}}$ would promote conversion to O-Mad2 because of $\beta 1$ 's role in stabilizing O-Mad2 (refs 19,22,23,26). The tendency of the TRIP13 hexamer to disassemble 18, especially in the absence of $\mathrm{p} 31$-substrate, may provide a mechanism to release Mad2 ${ }^{\mathrm{NT}}$. Working on the estimate that 8-10 ATP molecules are hydrolysed for each C-Mad2 molecule converted 17, the C-Mad2 conformational transition would involve unwinding of no more than the aA helix. 
The p31-substrate stimulates TRIP13 ATPase activity 17, indicating it promotes an activating conformational change of TRIP13. The conformational differences in the TRIP13p31-substrate complex between classes 2 and 3 are suggestive of class 3 as an activated state, primed for ATP hydrolysis. Compared with class 2, TRIP13 monomers D to F have changed their orientations relative to monomers $\mathrm{A}$ to $\mathrm{C}$, with the conformational change progressively increasing from D to F resulting in their shift towards the p31-substrate (Fig. 5a and Supplementary Video4). These changes in TRIP13 are accompanied by a small rotation of $\mathrm{p} 31^{\text {comet: }} \mathrm{C}-\mathrm{Mad} 2$ so that $\mathrm{p} 31^{\text {comet }}$ itself moves closer to TRIP13 monomers D and E. Monomer F shows the largest displacement towards the p31-substrate module, allowing its pore loop-2 to approach Mad2 ${ }^{\mathrm{NT}}$ (Fig. 5a,b). The effect of the p31-substrateinduced conformational change in TRIP13 is to move monomers $\mathrm{E}$ and $\mathrm{F}$ along the axis of translocation.

On conversion of O-Mad2 to C-Mad2 the $\beta 2-3$ hairpin rearranges. Mutations of the $\beta 2-3$ hairpin suppress this conversion 27. In the activated state, the approaching F monomer shifts the $\beta 2-3$ hairpin towards aA, perturbing its interactions with the C-Mad2 core (Fig. 5b). Thus, an interesting possibility is that the energy of p31-substrate binding to TRIP13 is converted into torsional energy within the TRIP13 ring that destabilizes C-Mad2 core interactions with the $\beta 2-\beta 3$ hairpin.

The proposed TRIP13-catalysed rotary motion of the globular region of the $\mathrm{p} 31^{\text {comet:}} \mathrm{C}$ Mad2 relative to the fixed Mad2 ${ }^{\mathrm{NT}}$ presents a mechanism to explain how local unwinding of a secondary structural element remodels tertiary and quaternary structure (Fig. 5c).

\section{Methods}

\section{Cloning, expression and purification of TRIP13 complexes}

The codon optimized DNA coding sequences (CDSs) of full-length human TRIP13 (Q15645) and $\mathrm{p} 31^{\text {comet }}(\mathrm{Q} 15013)$ were cloned individually into the bacterial pETM11 expression vector with an N-terminal TEV protease-cleavable $\mathrm{His}_{6}$ tag. Mad2 (Q13257) CDS was cloned into a custom modified version of the pETM11 vector where the Nterminal $\mathrm{His}_{6}$ tag was replaced by a C-terminal $3 \mathrm{C}$-cleavable $\mathrm{His}_{6}$ tag. QuickChange and Gibson cloning respectively generated the mutant versions of TRIP13, Mad2 and $\mathrm{p} 31^{\text {comet }}$. These include TRIP13: E269A plus D272A and E269R plus D272R, E253Q; Mad2: L5S6R7 to L5E6E7; and the N-terminal 7 and 9 residue deletions; p31 ${ }^{\text {comet: deletion of }}$ R99, K100, K110, K111, K112, R122, K123 and K270A (TRIP13-binding site on monomer E). Proteins were individually expressed in the BL21 Star (DE3) E. coli strain at $18{ }^{\circ} \mathrm{C}$ for $12 \mathrm{~h}$. TRIP13, p31 $1^{\text {comet }}$ and Mad2 were individually purified by Ni-NTA based protein purification (Ni-NTA Agarose resin) followed by either TEV or 3C protease cleavage and gel filtration (Superdex 75 10/300 column). TRIP13 and p31 ${ }^{\text {comet }}$ were further purified on a $6 \mathrm{~mL}$ Resource Q column (GE Healthcare Life Sciences) prior to size exclusion chromatography. MCC and $\mathrm{APC} / \mathrm{C}^{\mathrm{MCC}}$ complex cloning, expression and purification were described in 28 . 


\section{C-Mad2 complex remodelling experiments}

Purified $\mathrm{p} 31^{\text {comet }}$ and either wild type or mutant Mad2 were incubated with purified maltose-binding protein (MBP)-Cdc20 (Q13257) (ref. 28) for $2 \mathrm{~h}$ at $23{ }^{\circ} \mathrm{C}$. The resulting p31 ${ }^{\text {comet }}$ :C-Mad2:MBP-Cdc20 complex was purified by gel filtration using a Superdex 200 $10 / 300$ column. For disassembly of either the p31 $1^{\text {comet }}: \mathrm{C}-\mathrm{Mad} 2$ :MBP-Cdc20 complex or the MCC (both the MCC alone and MCC of the APC/C ${ }^{\mathrm{MCC}}$ complex) $10-15 \mu \mathrm{Mols}$ of complex were incubated with three-fold molar excess of TRIP13 as either wild type or the Q253E mutant (accounting for hexamerization of TRIP13), in a disassembly buffer (50 mM HEPES pH 8.0, $10 \mathrm{mM} \mathrm{MgCl}_{2}$ and $3 \mathrm{mM} \mathrm{ATP)} \mathrm{in} \mathrm{a} \mathrm{total} \mathrm{volume} \mathrm{of} 50 \mu \mathrm{L}$. In the MCC and $\mathrm{APC} / \mathrm{C}^{\mathrm{MCC}}$ containing reactions, a 10 -fold excess of $\mathrm{p} 31^{\text {comet }}$ was also added. Following incubation at $23{ }^{\circ} \mathrm{C}$ for $10 \mathrm{~min}$ the disassembly reaction was injected into a ÄKTAmicro (GE Healthcare) with a running buffer of $20 \mathrm{mM}$ HEPES pH 8.0, $300 \mathrm{mM} \mathrm{NaCl}, 10 \mathrm{mM}$ $\mathrm{MgCl}_{2}$ and $0.5 \mathrm{mM}$ TCEP. O-Mad2 eluted in a later fraction than the complexes of C-Mad2 with either $\mathrm{p} 31^{\text {comet }}$ or with both p31 ${ }^{\text {comet }}$ and MBP-Cdc20 (Extended Data Fig. 2a). OMad2 fractions were loaded (with input material) onto an SDS-PAGE gel and detected with a Western blot anti-Mad2 (ab10691; Abcam). TRIP13, Cdc20 and p31 ${ }^{\text {comet }}$ were detected with anti TRIP13 (sc-514285; Santa Cruz Biotechnology), Cdc20 (sc-8358; Santa Cruz Biotechnology) and p31 comet (MABE451; Merck) respectively.

To test MCC dissociation into C-Mad2:Cdc20 and BubR1:Bub3 (BubR1: O60566; Bub3:O43684) sub-complexes by (i) the combined action of TRIP13 ${ }^{\mathrm{E} 253 \mathrm{Q}}$-and $\mathrm{p} 31^{\text {comet }}$, (ii) TRIP13 ${ }^{\mathrm{E} 253 \mathrm{Q}}$ alone and (iii) $\mathrm{p} 31^{\text {comet }}$ alone, $30 \mu \mathrm{Mols}$ of MCC was mixed with (i) a 1.1-fold excess of TRIP13 ${ }^{\mathrm{E} 253 \mathrm{Q}}$ hexamer and a 10-fold excess $\mathrm{p} 31^{\text {comet }}$, ii) a 1.1-fold excess of TRIP13 ${ }^{\text {E253Q }}$ and (iii) a 10-fold excess p31 ${ }^{\text {comet }}$ in buffer: $50 \mathrm{mM}$ HEPES pH 8.0, $10 \mathrm{mM}$ $\mathrm{MgCl}_{2}$ and $3 \mathrm{mM}$ ATP. The mixture was injected into a Superdex 200 10/300 gel filtration column fitted to an ÄKTAmicro (GE Healthcare) with a running buffer of $20 \mathrm{mM}$ HEPES $\mathrm{pH}$ 8.0, $300 \mathrm{mM} \mathrm{NaCl}, 10 \mathrm{mM} \mathrm{MgCl}$ and $0.5 \mathrm{mM}$ TCEP and ATP. Peak eluted fractions were analysed using Coomassie-stained gels.

\section{Reconstitution of TRIP13 ${ }^{\text {p31-substrate }}$ complex for EM analysis}

A ternary complex composed of $\mathrm{p} 31^{\text {comet }}$ :C-Mad2:Cdc20 was purified by Ni-NTA column chromatography starting with a co-lysate of C-Mad2:MBP-Cdc20 co-expressing Hi-5 insect cells 28 with p31 ${ }^{\text {comet }}$ expressing BL21 Star (DE3) cells, followed by TEV cleavage and gel filtration (Superdex 200 10/300 column). Purified TRIP13 Q253E was incubated with equimolar amounts of the $\mathrm{p} 31^{\text {comet }}: \mathrm{C}-\mathrm{Mad} 2: \mathrm{Cdc} 20$ complex (accounting for hexamerization of TRIP13) in an assembly reaction buffer (50 mM HEPES pH 8.0, $10 \mathrm{mM} \mathrm{MgCl}_{2}$ and 5 $\mathrm{mM}$ ATP $\gamma \mathrm{S}$ ) for $45 \mathrm{~min}$ at $23{ }^{\circ} \mathrm{C}$. The $50 \mu \mathrm{L}$ assembly reaction was injected into a Superdex 200 5/150 GL column fitted to an ÄKTAmicro (GE Healthcare) with a running buffer containing $20 \mathrm{mM}$ HEPES pH 8.0, $300 \mathrm{mM} \mathrm{NaCl}, 10 \mathrm{mM} \mathrm{MgCl}_{2}, 0.5 \mathrm{mM}$ TCEP and 0.3 $\mathrm{mM}$ ATP $\gamma \mathrm{S}$. The complex eluted in a peak fraction at a concentration of $0.3 \mathrm{mg} / \mathrm{mL}$ and was used to prepare cryo-EM grids.

\section{Electron microscopy}

$2.5 \mu \mathrm{l}$ of the TRIP13 3 31-substrate complex eluted fraction was applied to Quantifoil Holey carbon R1.2/1.3 Au 300 grids, treated with a 9:1 argon:oxygen plasma for $30 \mathrm{~s}$ before use. 
The grids were incubated for $30 \mathrm{~s}$ at $4{ }^{\circ} \mathrm{C}$ and $100 \%$ humidity and then blotted for $6 \mathrm{~s}$ and plunged into liquid ethane using an FEI Vitrobot III. Specimens were imaged using both EPU software (FEI) and Serial EM (Mastronarde Group) at a nominal magnification of 81,000 , yielding a pixel size of $1.43 \AA$ Apixel at specimen level on an FEI Titan Krios electron microscope operating at $300 \mathrm{kV}$ accelerating voltage. Zero-energy-loss micrographs were recorded using a Gatan K2-Summit direct electron detector executed in counting mode at the end of a Gatan GIF-Quantum energy filter with a slit width of $20 \mathrm{eV}$. Images were collected at a dose rate of $\sim 2.6$ electrons $/ \AA^{2} / \mathrm{s}$. Exposures of 16 seconds were dose-fractionated into 20 movie frames with a total dose of $\sim 41$ electrons per $\AA^{2}$. Defocus values in the final data set ranged from -2.0 to $-3.6 \mu \mathrm{m}$.

\section{Image processing}

Movie frames were aligned using motioncorr2 (ref. 29) before subsequent processing. Contrast transfer function parameters were calculated using Gctf 30. Particles in 140x140 pixels were selected automatically by using Gautomatch (Kai Zhang) with an inter-particle distance cutoff of $150 \AA$. The initial 2D references for the automated picking were created by manual picking. Picked particles were extracted and processed in RELION 2.1 (ref. 31). After three rounds of 2D classification where 2D classes with poor structural features were removed with 30-40\% of particle classes finally selected for 3D refinement. The initial model for 3D refinement was generated $a b$ initio using the random start-up procedure in IMAGIC-4D 32. Overall 3D refinement yielded a 3D reconstruction with $4.5 \AA$ resolution. To further improve the EM density map of TRIP13, a soft mask including the most rigid TRIP13 monomers A/B/C/D (TRIP13 ${ }^{\mathrm{A} / \mathrm{B} / \mathrm{C} / \mathrm{D}}$ ) was used for local alignment during refinement. This improved the resolution to $4.3 \AA$ for TRIP13 $\mathrm{A} / \mathrm{B} / \mathrm{C} / \mathrm{D}$. All resolution estimations were from gold-standard FSC calculations to avoid over-fitting and reported resolutions are based on the FSC $=0.143$ criterion 33. Final FSC curves and sharpening of density maps was performed as in 31 .

3D classification with a global search and a sampling angular interval of $7.5^{\circ}$ allowed the identification of apo (class 1: 20\% of total) and p31-substrate bound (80\% of total) TRIP13 complexes. The TRIP13:p31-substrate class was further classified yielding the TRIP13:p31substrate basal (class 2: 22\%), the TRIP13:p31-substrate activated states (class 3: 24\%) and a reconstruction (34\%) with resemblance to the TRIP13:p31-substrate basal state but that featured poorer alignment accuracy.

\section{Map Visualization}

Figures and vidoes were generated using the PyMOL (Molecular Graphics System, 2.0.3 Schrodinger, LLC) and Chimera 34.

\section{Model building}

Initial fitting and superposition of human TRIP13 (ref. 18) and p31 comet:C-Mad2:MBP 19 were performed with Chimera 34. Model building and flexible fitting were performed in COOT 35. TRIP13 pore loops- 1 and -2 model building was performed $a b$ initio using the crystal structure of the PAN regulatory particle 36 as an initial reference using our highest resolution maps. The TRIP13 ${ }^{\text {loop } 111-121}$ was built as an idealized polyAla chain fitting to 
the corresponding density. The $\mathrm{p} 31^{\text {comet }} \mathrm{a} 3-4$ loop was built as polyAla chain fitting the corresponding density of a TRIP13:p31-substrate map obtained after focussed classification of the N-terminal domain of TRIP13 monomer $\mathrm{E}$ with additional guidance from published cross-linking data restraints 18 . The Mad2 N-terminus ( $\operatorname{Mad}_{2}{ }^{\mathrm{NT}}$ ) was built by flexible fitting of Mad2 (ref. 25). Model refinement of the TRIP13:p31-substrate structure was performed with PHENIX 37 using 3D classes 2 and 3.

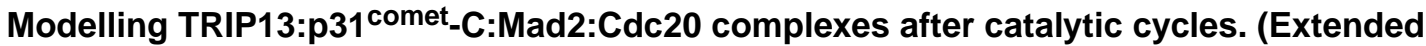 Data Fig. 9 and Supplementary Videos 2 and 3)}

To model TRIP13 after the first catalytic cycle (i.e. basal state 1), we superimposed monomer $\mathrm{B}$ onto monomer $\mathrm{A}$ (thus new $\mathrm{A}^{1}$ was formerly $\mathrm{B}^{0}$, new $\mathrm{F}^{1}$ was formerly $\mathrm{A}^{0}$ and new $\mathrm{E}^{1}$ was $\mathrm{F}^{0}$ etc. [where superscript ' 0 ' denotes the pre-catalytic state and superscript ' 1 ' denotes the first post-catalytic state]). C-Mad2 ${ }^{\mathrm{NT}}$ (here defined as residues 2 to 12) was modelled based on its conformation in the pre-catalytic conformation (i.e. unchanged). The globular portion of $\mathrm{p} 31^{\text {comet. }} \mathrm{C}-\mathrm{Mad} 2$ (all residues of $\mathrm{p} 31^{\text {comet }}$ and residues 13 to 205 of CMad2) were re-positioned onto the new $\mathrm{C}^{1}-\mathrm{D}^{1}$ interface (in effect maintaining the same contacts with the shifted $\mathrm{D}^{0}-\mathrm{E}^{0}$ subunits) to avoid clashes with the repositioned $\mathrm{E}^{1}$ and $\mathrm{F}^{1}$.

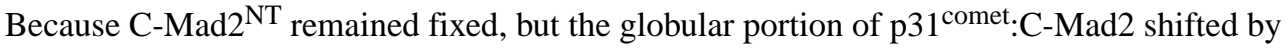
$7.6 \AA$ (due to the vertical movement and $60^{\circ}$ rotation (Extended Data Fig. 9i)), the $\mathrm{Ca}$ atoms of Thr12 of C-Mad2 $2^{\mathrm{NT}}$ and Leu13 the globular portion of C-Mad2 were separated by $11 \AA$ (Extended Data Fig. 9h). Reconnecting Thr12 and Leu13 was performed by unwinding the first turn of the C-Mad2 aA helix (residues 12 to 17) with the coordinates refined in COOT 35. The same procedure was applied to remodel the TRIP13:p31 ${ }^{\text {comet:C-Mad2:Cdc20 }}$ complex basal state 2 (post catalytic cycle 2) (Supplementary Video 2). The calculation that one helical turn is unwound per catalytic cycle is based on the following reasoning:

The axial distance between adjacent residues in an a-helix $=1.5 \AA$.

The axial distance between adjacent residues in a $\beta$-sheet $=3.5 \AA$.

Thus the difference in axial distance between adjacent residues in an $\alpha$-helix and $\beta$-sheet is $2 \AA /$ residue. Stretching an a-helix by $7.6 \AA$ by forming an extended chain requires $7.6 \AA / 2$ $\AA /$ residue $=3.8$ residues $=1.06$ helical turn (assuming 3.6 residues/turn).

Structural conservation was calculated using CONSURF 38. The interface surface area

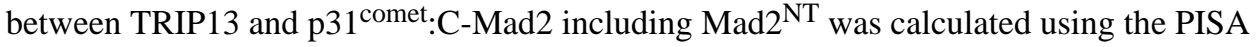
protein interface server 39. Protein sequence alignment was performed using Jalview 40. 


\section{Extended Data}

a
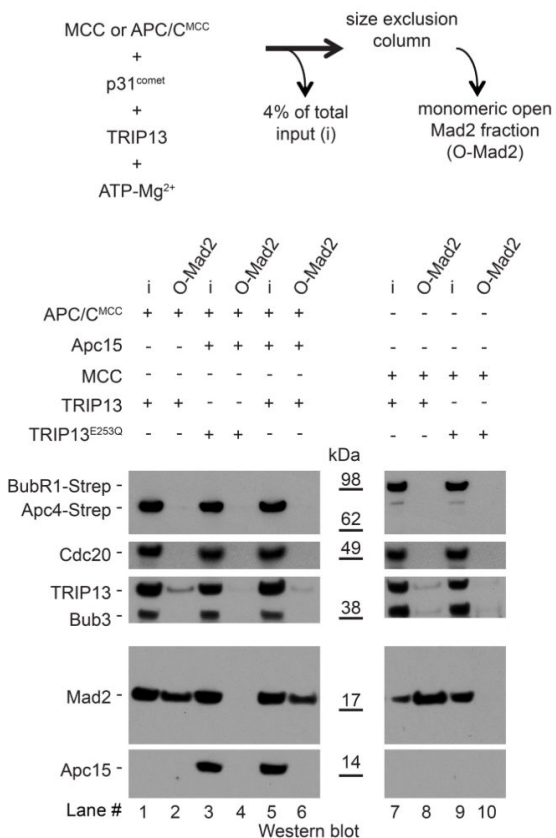

b

$M C C+p 31^{\text {comet }}+$ TRIP13

$M C C+p 311^{\text {comol }}+$ TRIP13 12530

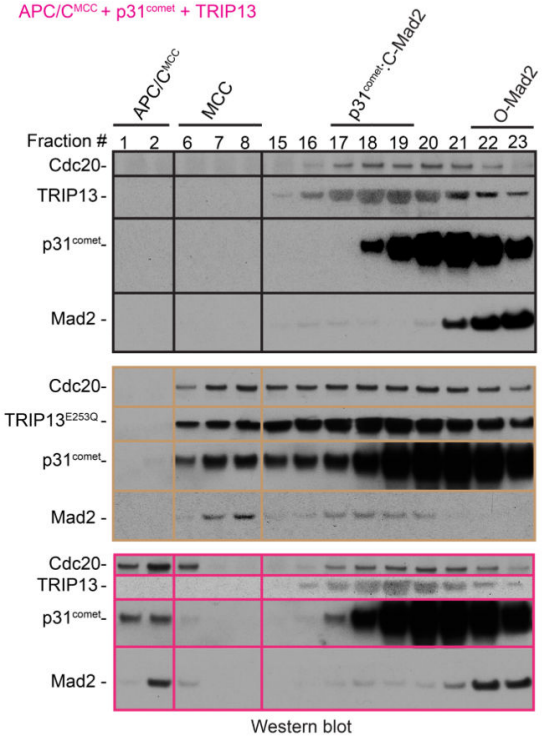

c

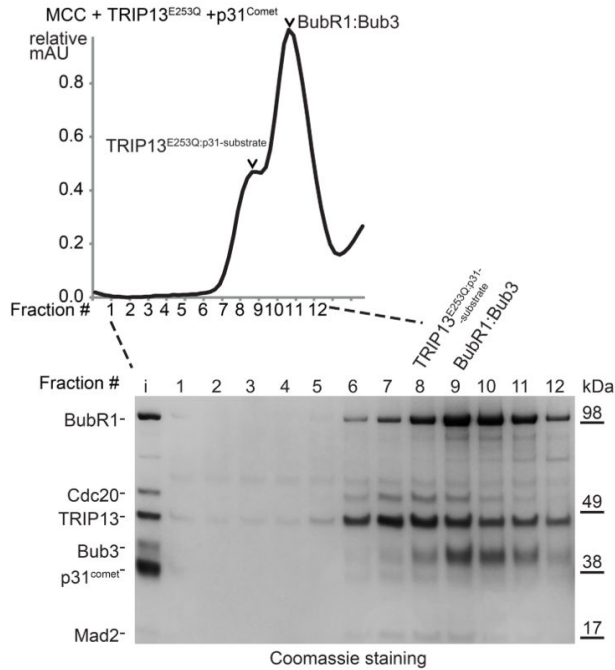

d
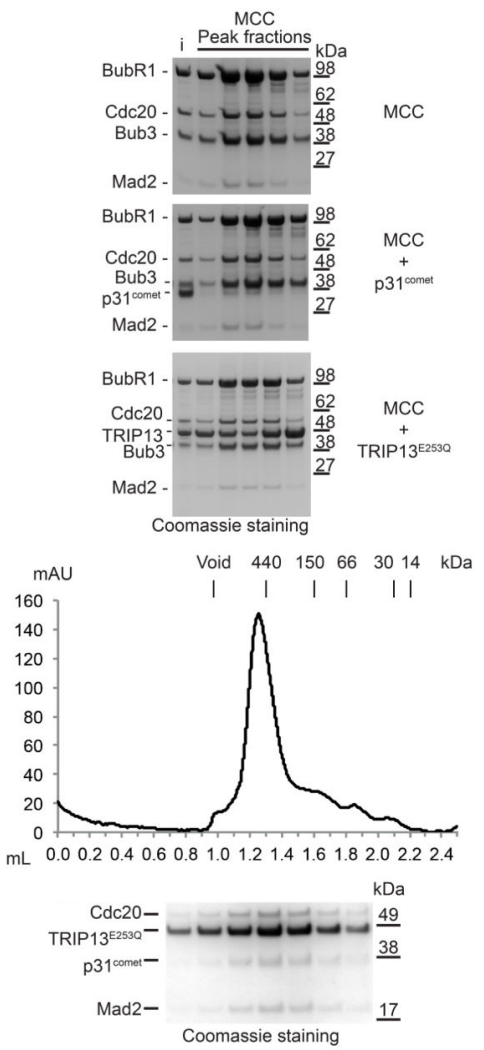

Extended Data Figure 1. Biochemical characterization of Mad2-containing complexes with TRIP13.

a, TRIP13 and p31 ${ }^{\text {comet }}$ can extract O-Mad2 from MCC and $\mathrm{APC} / \mathrm{C}^{\mathrm{MCC}}$ regardless of Apc15. Western blot showing the disassembly reactions together with the respective input material (i) of $\mathrm{APC} / \mathrm{C}$-bound $\mathrm{MCC}\left(\mathrm{APC} / \mathrm{C}^{\mathrm{MCC}}\right.$ ), in either the absence (lanes 1-2) or presence of Apc15 (lanes 3-6) and MCC alone (lanes 7-10) (experimental design is shown on top). Negative control reactions (lanes 3-4 and 9-10) were performed with the 
TRIP13 ${ }^{\mathrm{E} 253 \mathrm{Q}}$ mutant. Mad2 levels were detected with an anti-Mad2 antibody. Loading controls of Apc4-Strep, Apc15, Cdc20, TRIP13, Bub3 were detected with antibodies specific for Strep, Apc15, Cdc20, TRIP13 and Bub3 respectively. BubR1 (lanes 7-10) was detected with an anti-Strep antibody. $\mathbf{b}$, Western blots showing eluted size exclusion (Superdex 200 10/300 column) fractions of the MCC and Mad2 remodelling reactions catalysed by TRIP13:p31 $1^{\text {comet }}$ in the context of free MCC and APC/C ${ }^{\mathrm{MCC}}$. The fractions corresponding to (i) $\mathrm{APC} / \mathrm{C}^{\mathrm{MCC}}$, (ii) $\mathrm{MCC}$, (iii) $\mathrm{p} 31^{\text {comet }} \mathrm{C}-\mathrm{Mad} 2$ and (iv) monomeric C$\mathrm{Mad} 2$ are shown. A reference gel for size exclusion column elution fractions corresponding to $\mathrm{p} 31^{\text {comet. }} \mathrm{C}-\mathrm{Mad} 2$ :Cdc20-MBP, p31 ${ }^{\text {comet. }} \mathrm{C}-\mathrm{Mad} 2$ and monomeric C-Mad2 is shown in Extended Data Fig. 2a. c, Analysis of TRIP13-p31 ${ }^{\text {comet }}$ complexes with the MCC using size exclusion chromatography in the presence of ATP. Coomassie-stained gel showing the gel

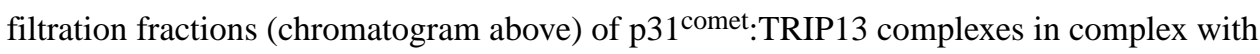
MCC (fraction 8 is the p31 $1^{\text {comet }}$ TRIP13 complex with C-Mad2-Cdc20 and fraction 9 is the BubR1:Bub3 complex). Input material (i) is shown on the left. d, MCC binds p31 ${ }^{\text {comet }}$ and not TRIP13 alone. Coomassie-stained gel showing the gel filtration fractions of the MCC (top gel) and in the presence of p31 $1^{\text {comet }}$ (middle gel) and TRIP13 ${ }^{\mathrm{E} 253 \mathrm{Q}}$ (lower gel). e, Chromatogram (top) and SDS-PAGE (bottom) of the gel filtration performed with the TRIP13 ${ }^{\mathrm{E} 253 \mathrm{Q}}: \mathrm{p3} 1^{\text {comet:}} \mathrm{C}-\mathrm{Mad2}$ :Cdc20 complex in the presence of ATP $\gamma \mathrm{S}$. Experiments in a-e were performed in triplicate with similar results. See Supplementary Fig. 1 for gel source data. 


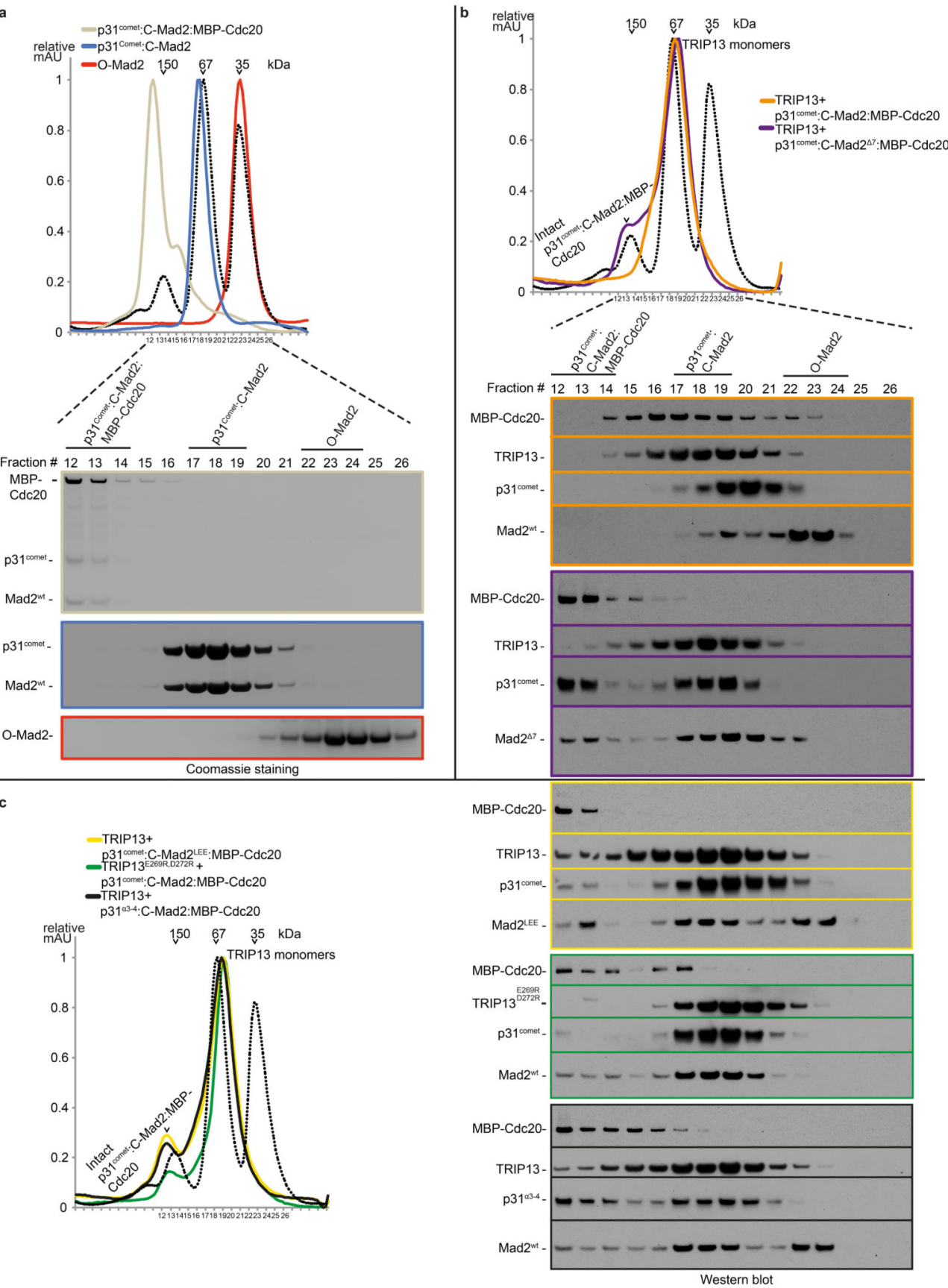

Extended Data Figure 2. Biochemical assay for TRIP13:p31 ${ }^{\text {comet }}$-catalysed O-Mad2 generation. Shown are size exclusion (Superdex 200 10/300 column) chromatograms and corresponding Coomassie-stained gels for the Mad2 remodelling reaction catalysed by TRIP13:p31 ${ }^{\text {comet }}$. a,

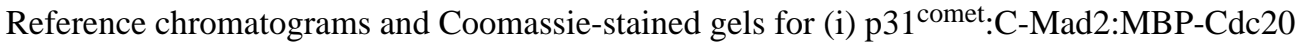
(brown trace), (ii) p31 comet:C-Mad2 (blue trace) and (iii) monomeric O-Mad2 (red trace). Chromatograms and gels are colour-coded. Monomeric O-Mad2 elutes in fractions 22-24,

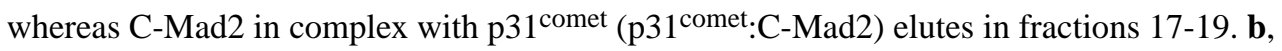
Chromatograms and Coomassie-stained gels for the products of the reaction of TRIP13 with 
(i) wild type $\mathrm{p} 31^{\text {comet }}$ and Mad2 (orange trace) and (ii) WT p31 $1^{\text {comet }}$ and mutant C-Mad2 (C-Mad2 ${ }^{\Delta 7}$ (7 N-terminal residues deleted)). c, Chromatograms and Coomassie-stained gels for the products of the reaction with (i) mutant C-Mad2 $2^{\mathrm{LEE}}$, wild type TRIP13 and $\mathrm{p} 31^{\text {comet }}$ (yellow trace), (ii) mutant TRIP13 ${ }^{\mathrm{E} 269 \mathrm{R}, \mathrm{D} 272 \mathrm{R}}$ and wild type p31 ${ }^{\text {comet }}$ and C-Mad2 (green trace) and (iii) mutant p31 $1^{\text {comet-a } 3-4}$ and wild type TRIP13 and C-Mad2 (black trace).

Experiments in a-c were performed in triplicate with similar results. See Supplementary Fig. 1 for gel source data.

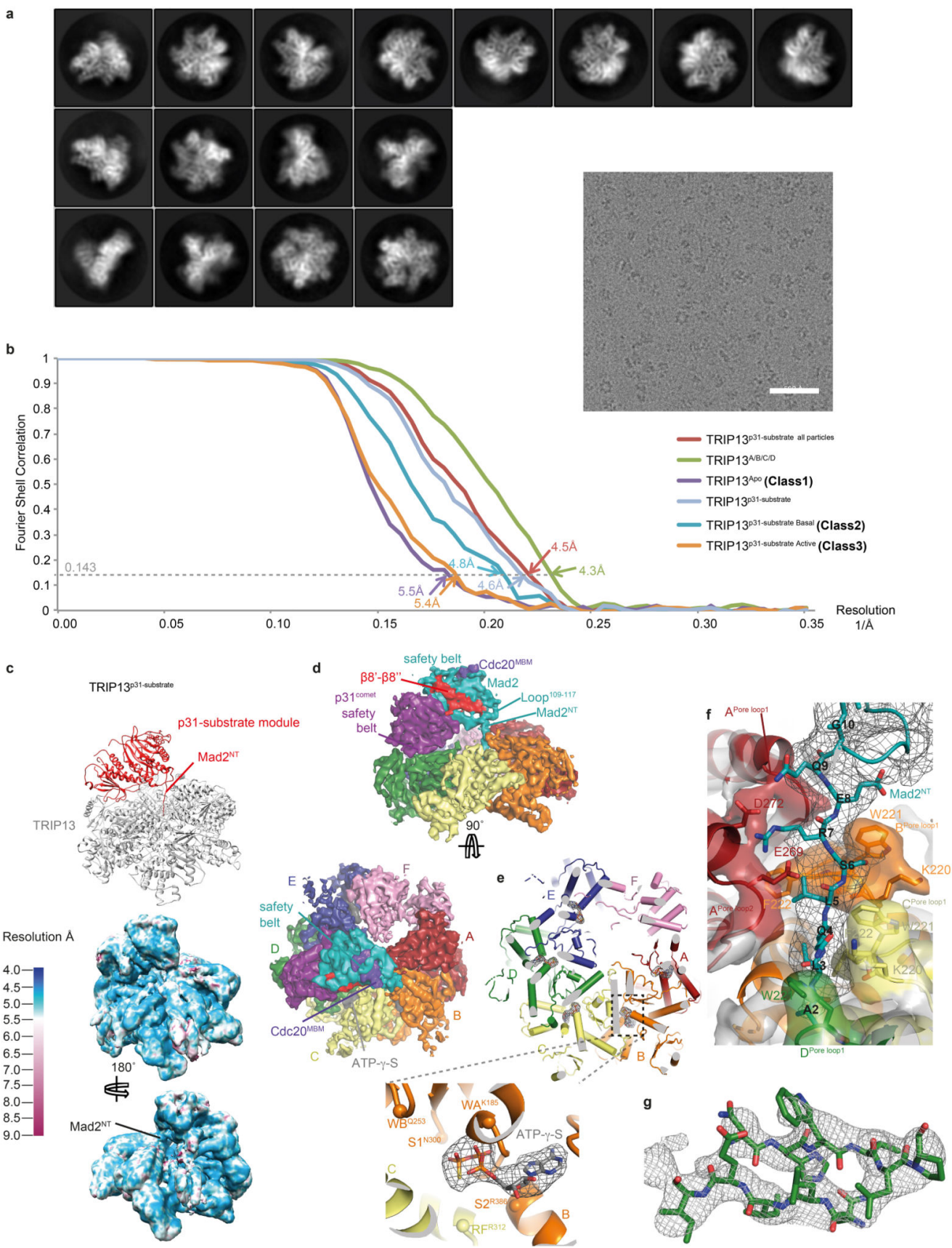

Nature. Author manuscript; available in PMC 2019 January 04. 
Extended Data Figure 3. Cryo-EM analysis and resolution of TRIP13 complexes in this study. a, Left: Gallery of two-dimensional class averages of TRIP13-p31-substrate showing different views representative of 50 two-dimensional classes. Right: A typical cryo-EM micrograph of TRIP13-p31-substrate representative of 3,630 micrographs. b, Fourier shell correlation (FSC) curves are shown for all the cryo-EM reconstructions determined in this study. c, local resolution maps calculated with RESMAP 41 of the TRIP13 3 31-substrate complex. d, Cryo-EM density of the TRIP13-p31-substrate reconstruction shown as in Fig. 1b. e, g, Representative density quality for the ATP $\gamma \mathrm{S}(\mathbf{e})$ and $\beta$-strand (g). In (e) critical residues for the TRIP13 catalytic site are indicated: WA (Walker A), WB (Walker B), S1 (sensor 1), S2 (sensor 2) and RF (Arg finger). f, Close up of the TRIP13 pore loops interacting with C-Mad2 ${ }^{\mathrm{NT}}$ from the cryo-EM density of the TRIP13-p31-substrate. EM density for C-Mad2 shown in black mesh, TRIP13 in transparent coloured surface. 


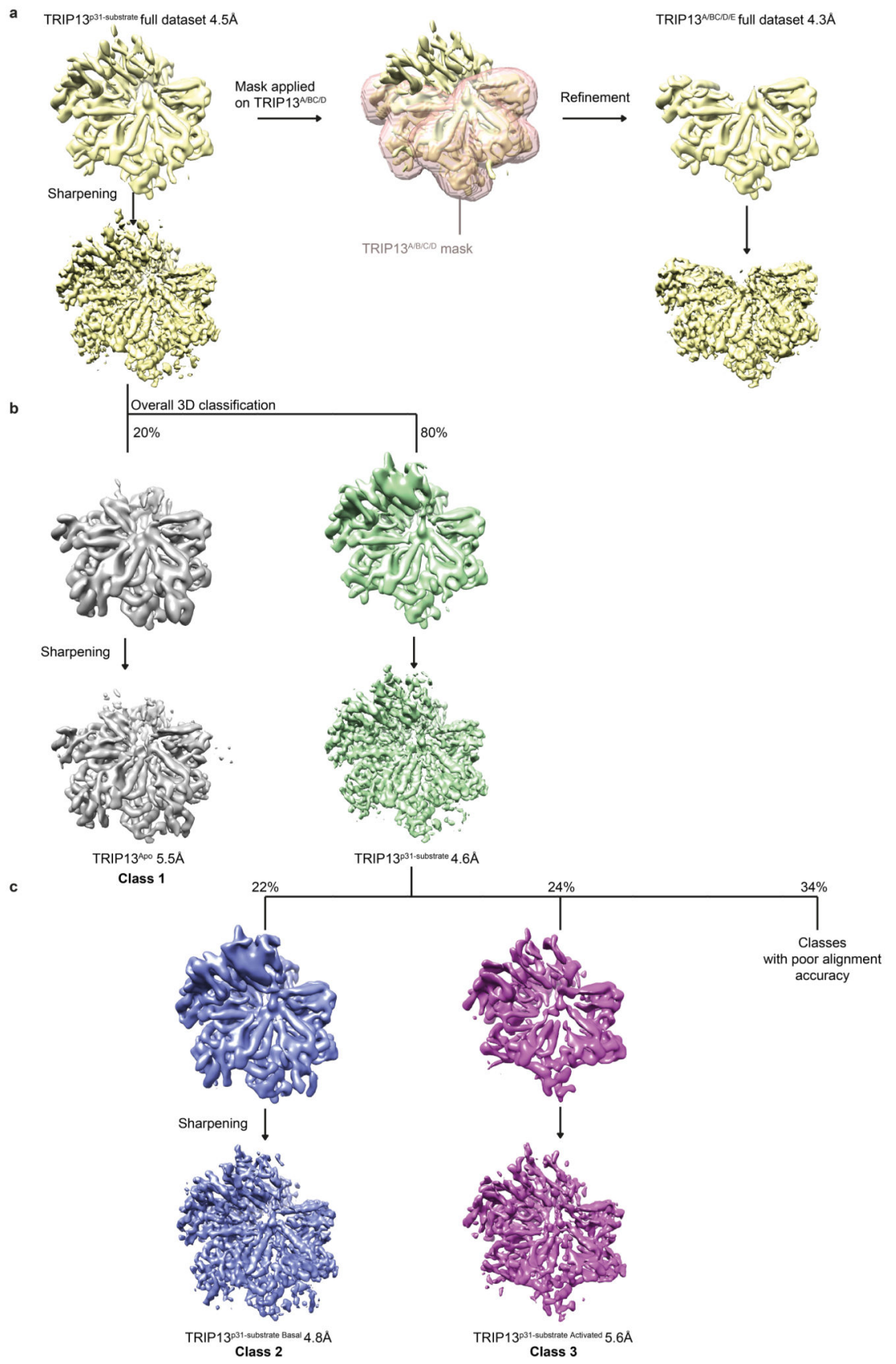

Extended Data Figure 4. Three-dimensional classification of TRIP13-p31-substrate full dataset. a, Local refinement (see Methods) by applying a mask covering TRIP13 1 /B/C/D/E monomers. b, c, 3D class averages obtained by classification (see Methods) of the TRIP13p31-substrate full dataset (b) and TRIP13-p31-substrate (c) are shown. The percentages relative to the total number of TRIP13-p31-substrate particles are shown. 
a
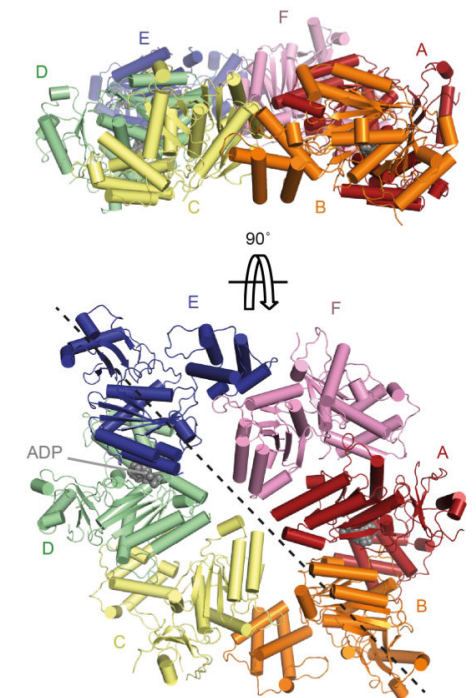

b
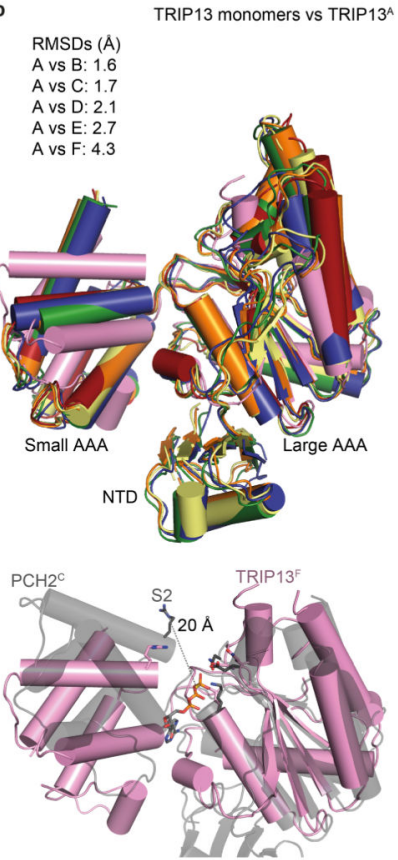
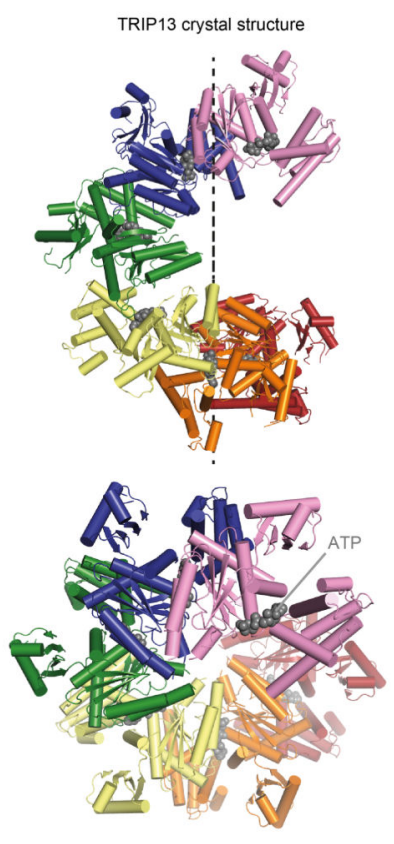
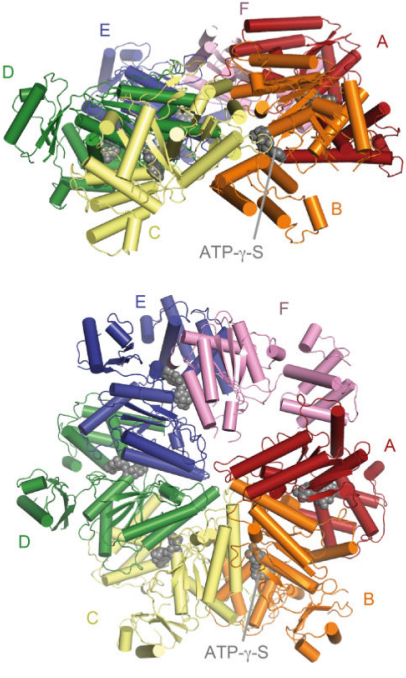
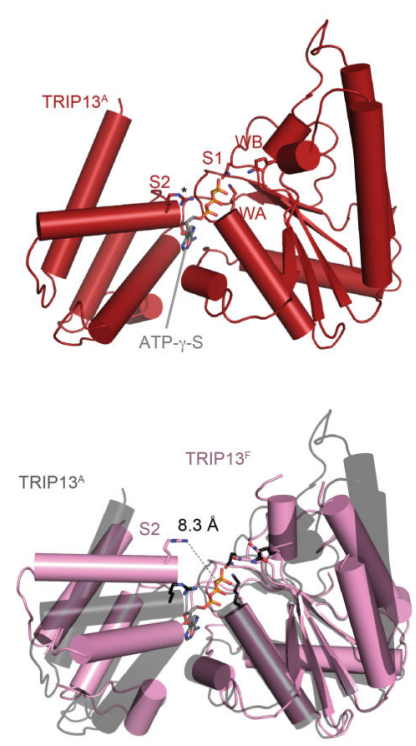
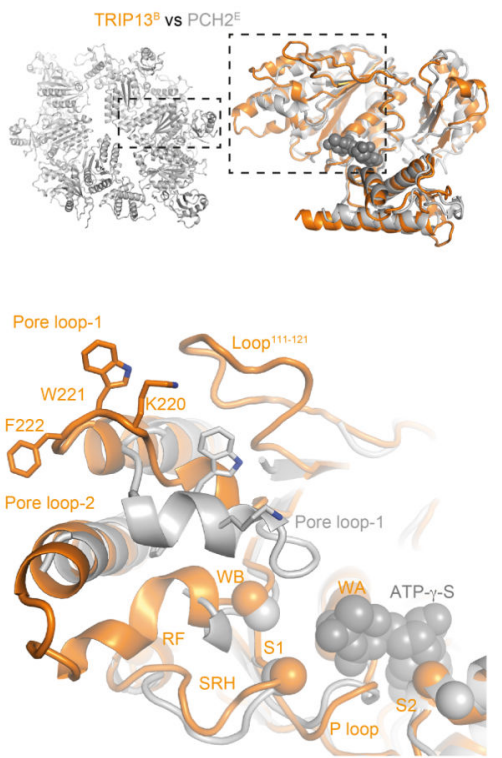

Extended Data Figure 5. Comparative analysis of TRIP13 structures.

a, Comparison of TRIP13 cryo-EM structure (right, this study) and previous TRIP13 crystal structures (left, $C$. elegans PCH2 (ref. 17), middle, human TRIP13 (ref. 17)). b, Comparison of TRIP13 monomers within the TRIP13 cryo-EM structure, RMSDs between TRIP13 ${ }^{\mathrm{A}}$ and other TRIP13 monomers are indicated in the insert table. A superimposition of all six TRIP13 monomers is shown, colour-coded according to Fig. 1 . TRIP13 ${ }^{\mathrm{F}}$ differs from all the other monomers in the relative orientation of the small and large AAA+ domains. Its conformation relative to TRIP13 ${ }^{\mathrm{A}}$ is shown in the lower right. The open conformation 
prevents nucleotide binding. (The sensor 2 residue (S2) is positioned too far from the ATPbinding site). Lower left panel shows a superimposition of TRIP13F onto an open subunit $\mathrm{C}$ (grey) of the PCH2 structure 17. c, Conformational differences in pore loop-1 between the PCH2:ADP 17 complex (grey) and the cryo-EM TRIP13:ATP $\gamma \mathrm{S}$ complex (orange) (this study).

a

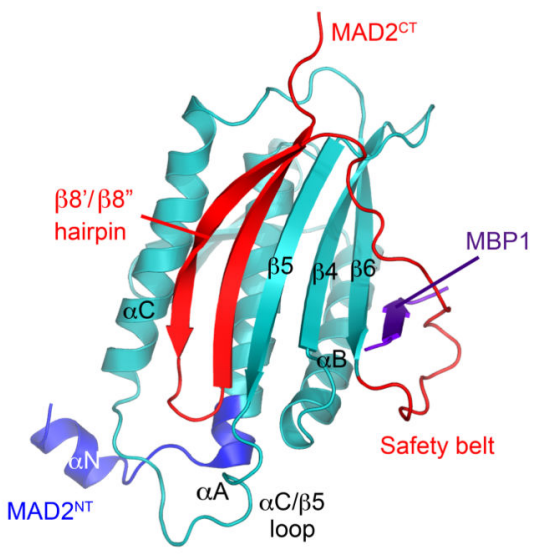

C-Mad2, PDB: 2v64

\section{d}

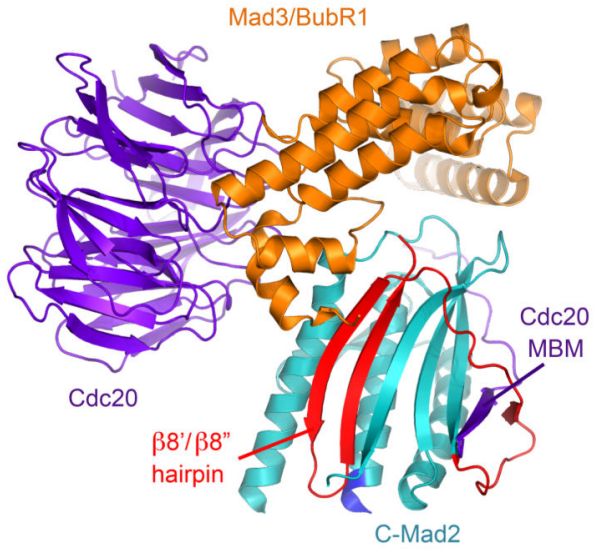

S. pombe mitotic checkpoint complex (MCC), PDB: 4aez

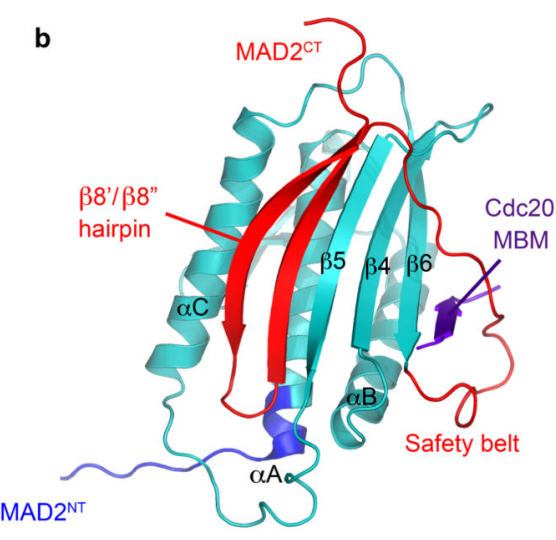

C-Mad2 (this work)

e

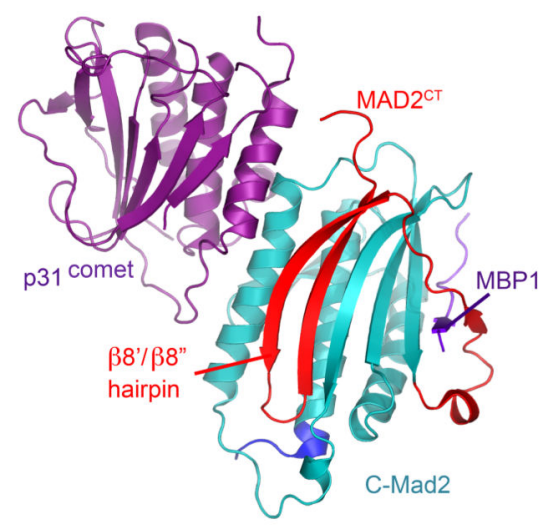

p31 comet $-C-M a d 2$, PDB: 2qyf c

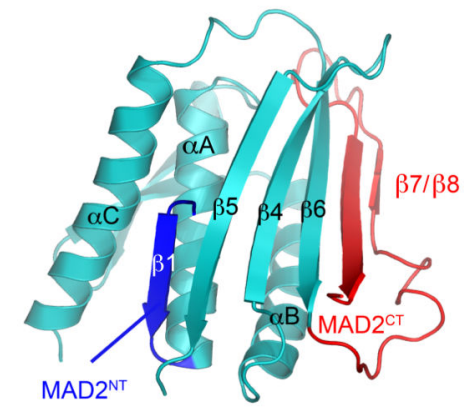

O-Mad2, PDB: 2v64

Extended Data Figure 6. Structures of Mad2.

a, C-Mad2 (ref. 23); b, in the TRIP13:p31 ${ }^{\text {comet. }}$ C-Mad2 complex (this work); c, O-Mad2 (ref. 23); d, the $S$. pombe mitotic checkpoint complex 42; e, p31 ${ }^{\text {comet.C-Mad2 complex } 19 .}$ In all figures the regions of Mad2 that reposition on the O-Mad2 to C-Mad2 transition are coloured blue and red for N-terminal (residues 1-16) and C-terminal (158 to 204) regions respectively. In O-Mad2 these are the $\mathrm{N}$-terminus $\left(\mathrm{Mad}_{2}{ }^{\mathrm{NT}}\right)$ and $\beta 1$ strand (blue) and $\mathrm{C}$ terminal $\beta 7-\beta 8$ hairpin (red). In C-Mad2 these are Mad2 ${ }^{\mathrm{NT}}$ including the $\alpha \mathrm{N}$ helix, and first turn of $\alpha \mathrm{A}$ (blue), and C-terminal $\beta 8^{\prime}-\beta 8^{\prime \prime}$ hairpin, safety belt and C-terminus ( $\mathrm{Mad}_{2}{ }^{\mathrm{CT}}$ ) (red). On conversion of O-Mad2 to C-Mad2, the $\beta 1$ strand is displaced and replaced by the $\beta 8$ '- $\beta 8$ ' hairpin. Residues $13-15$ of $\beta 1$ form an additional turn at the $\mathrm{N}$-terminus of $\alpha \mathrm{A}$ 
present in C-Mad2. The C-Mad2 ligand is coloured purple. MBM: Mad2-binding motif. MBP1: High affinity Mad2-binding peptide 43,44.
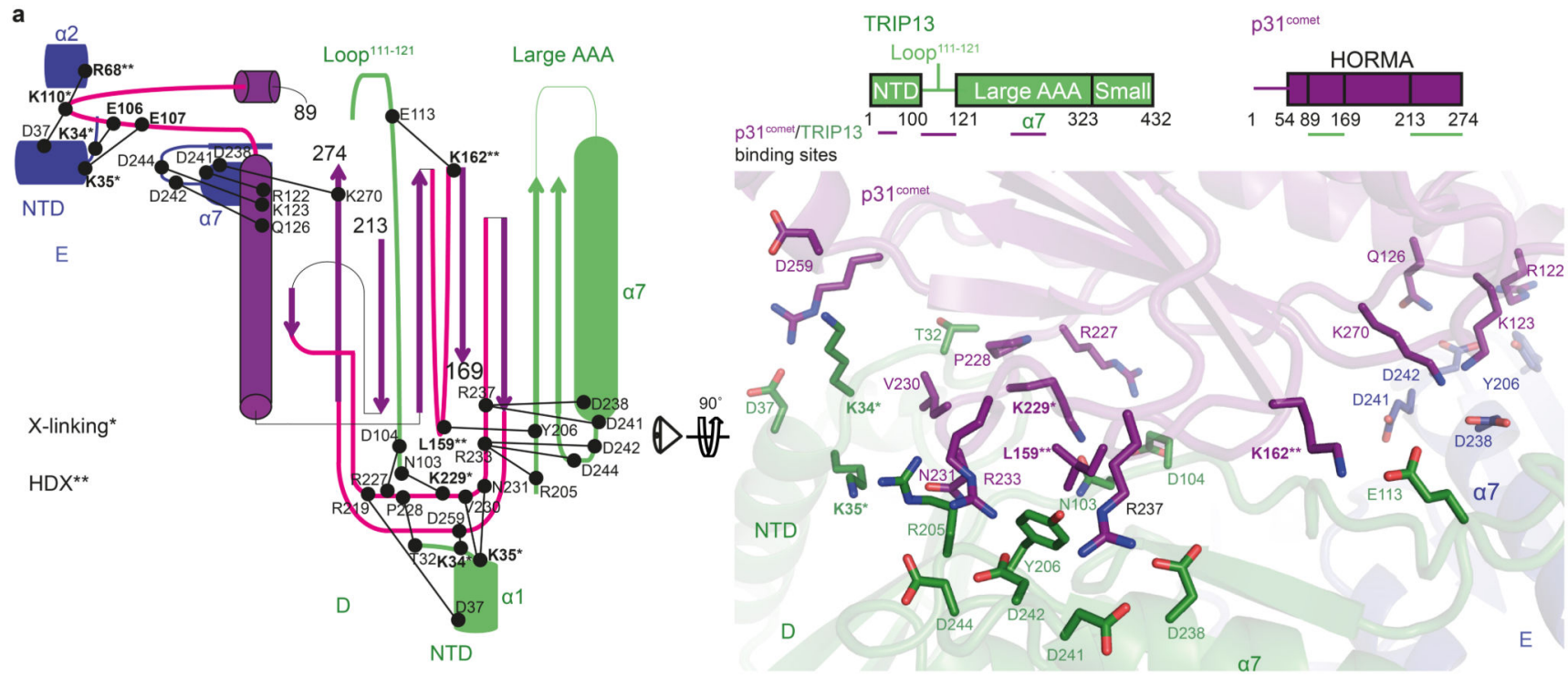

b

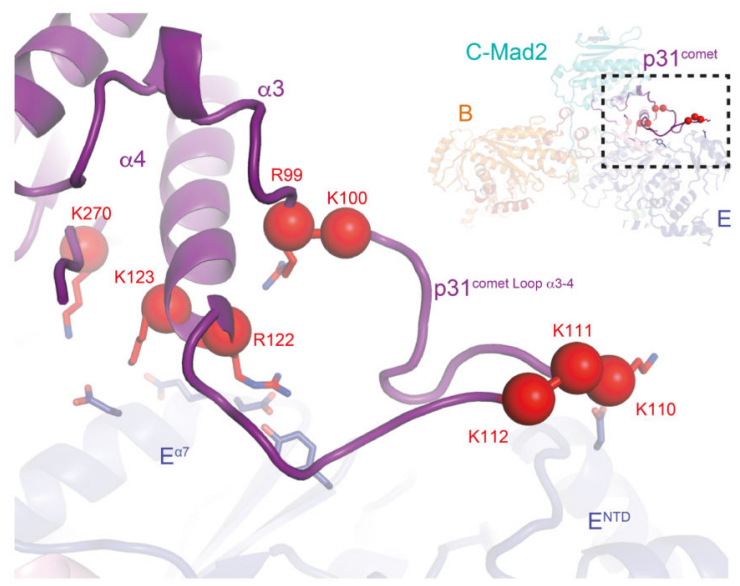

c

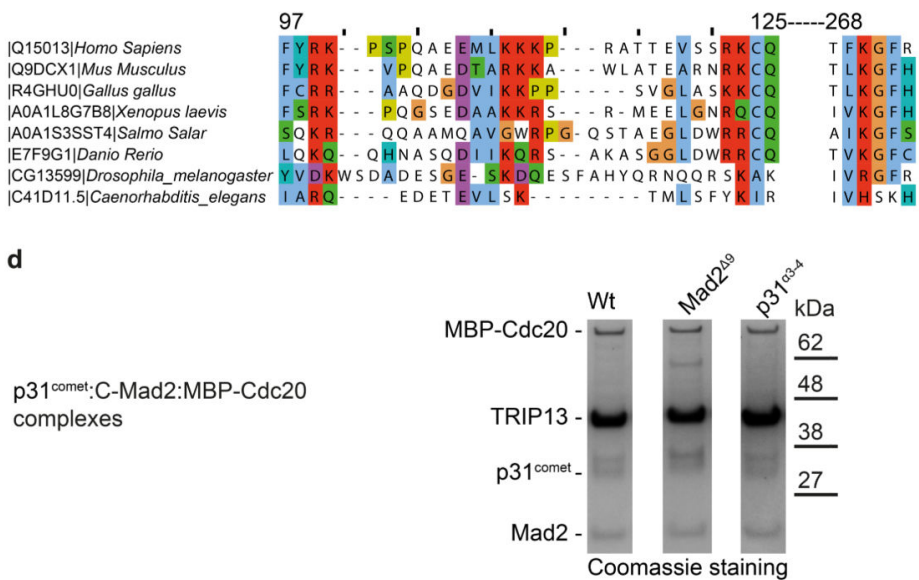

Extended Data Figure 7. TRIP13 interacts with p31 $^{\text {comet }}$ through a composite interface formed of monomers D and E.

a, Details of the interaction between TRIP13 and p31 ${ }^{\text {comet }}$. Left: schematic of TRIP13p31 comet interactions. Right: structure showing details of the main electrostatic contacts between TRIP13 and p31 ${ }^{\text {comet }}$. Above: Schematic of the domain architecture of TRIP13 and p3 $1^{\text {comet }}$. A row of aspartates on a 7 engages the conserved safety belt motif residues Arg233 and Arg237 of p31 comet. The adjacent Lys162 contacts a Glu-rich loop (111-121) in TRIP13 that is disordered in previous TRIP13 crystal structures 17,18 . In our structure the Glu-rich loop lies directly above pore loop-1. Glu104 and Asp105 of the TRIP13NTD_

ATPase domain linker, immediately preceding the Glu-rich loop, contact Arg227 and Lys 229 of the p31 ${ }^{\text {comet }}$ safety-belt, agreeing with the importance of Lys229 for TRIP13$\mathrm{p} 31^{\text {comet }}$ interactions in vitro 17 and in vivo 20. On monomer $\mathrm{E}$, the same acidic patch of a 7 
of the large AAA+ domain contacts basic residues at the N-terminus of a 3 of $\mathrm{p} 31^{\text {comet }} . \mathbf{b}$, Details of the interaction of the p31 ${ }^{\text {comet }}$ a 3-4 loop with TRIP13 subunit E. Seven basic residues shown were deleted and the mutant $\mathrm{p} 31^{\text {comet- } \mathrm{a} 3-4}$ was tested in Mad2 remodelling

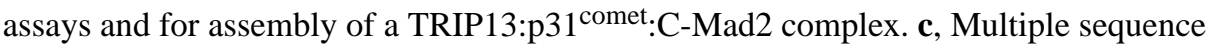
alignment of the p31 ${ }^{\text {comet }}$ a 3-4 loop. d, Deletion of the nine N-terminal residues of Mad2 $\left(\operatorname{Mad} 2^{\Delta 9}\right)$, and mutation of the $\mathrm{p} 31^{\text {comet }}$ a3-4 loop (p31 $1^{\text {comet-a3-4loop }) ~ d o ~ n o t ~ d i s r u p t ~}$

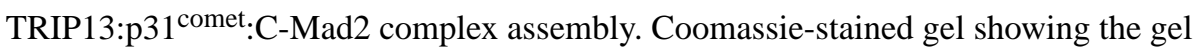
filtration fraction of wild type and relevant mutant TRIP13:p31 ${ }^{\text {comet:C-Mad2 complexes }}$ purified by size exclusion chromatography. Experiment in $\mathbf{d}$ was performed in triplicate with similar results. See Supplementary Fig. 1 for gel source data.

\section{a TRIP13A pore loop-1}

|Q15645|Homo sapiens

Q3UA06|Mus musculus |E1C6Q1|Gallus gallus |A0A1L8FWZ2|Xenopus laevis |A0A1S3QCD0|Salmo salar |F1RBS4|Danio rerio |Q8SX76|Drosophila melanogaster |Q09535|Caenorhabditis elegans

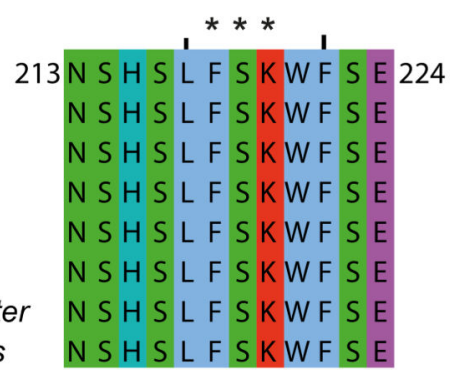

b TRIP13A pore loop-2

|Q15645|Homo sapiens |Q3UA06|Mus musculus |E1C6Q1|Gallus gallus |A0A1L8FWZ2|Xenopus laevis |A0A1S3QCD0|Salmo salar |F1RBS4|Danio rerio |Q8SX76|Drosophila melanogaster |Q09535|Caenorhabditis elegans

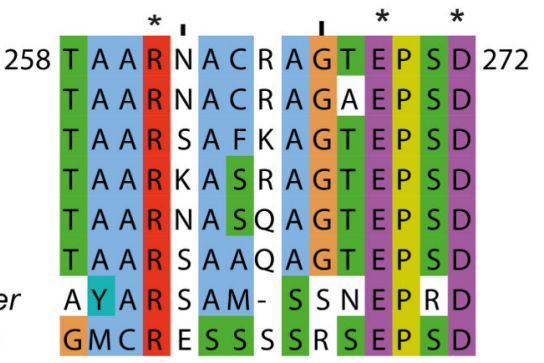

C Mad2 $^{\text {NT }}$

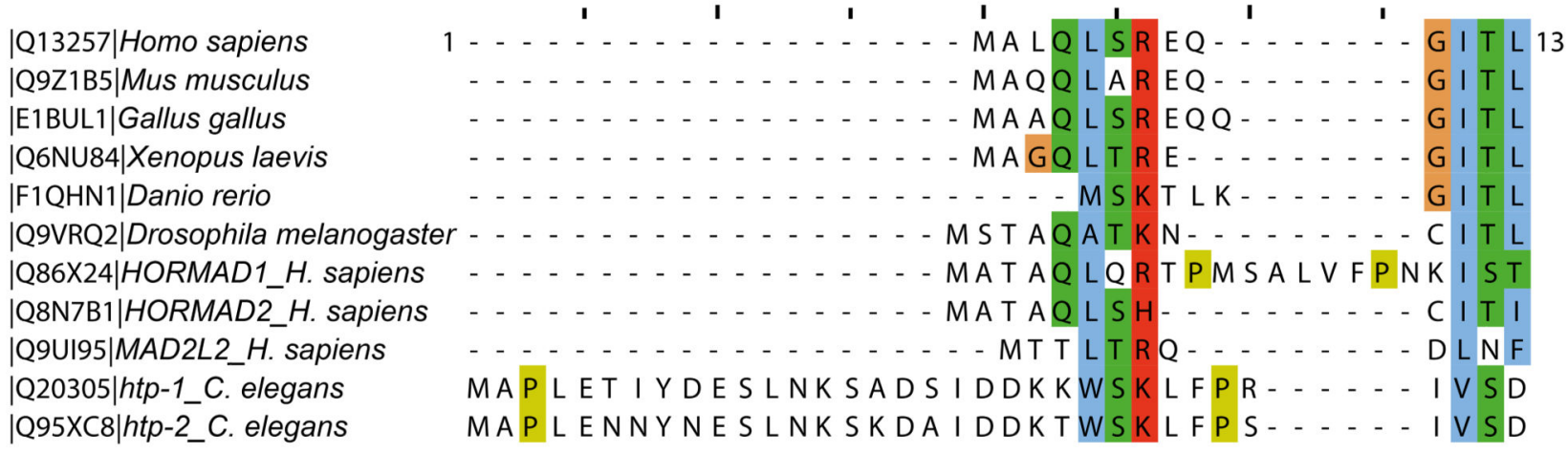

Extended Data Figure 8. Conservation of TRIP13 ${ }^{\text {pore loops }}$ and Mad2 $^{\text {NT }}$. 
Multiple sequence alignment of (a) TRIP13 pore loop-1 and (b) pore loop-2 and the (c) Nterminal region of Mad2.

a

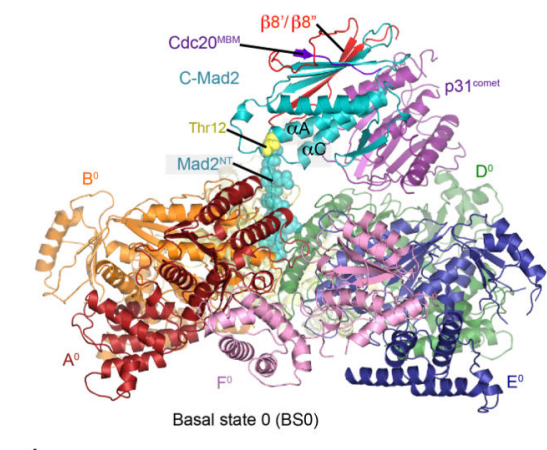

b

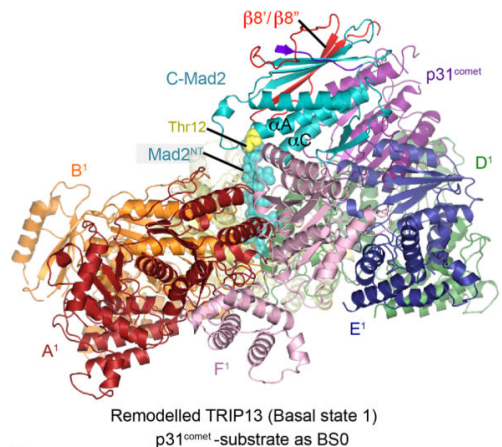

d
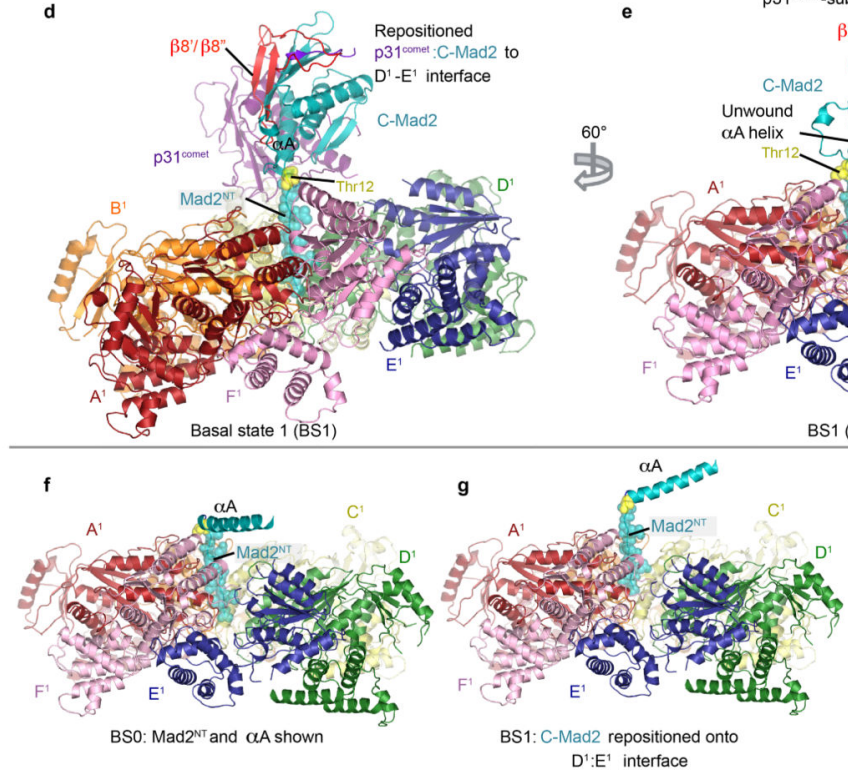

e

p31 comet-substrate as BSO
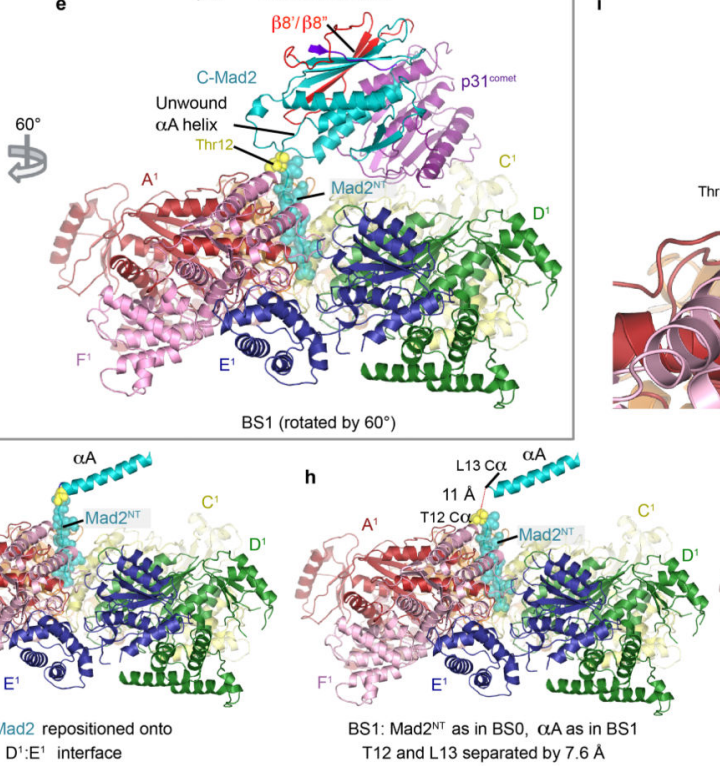
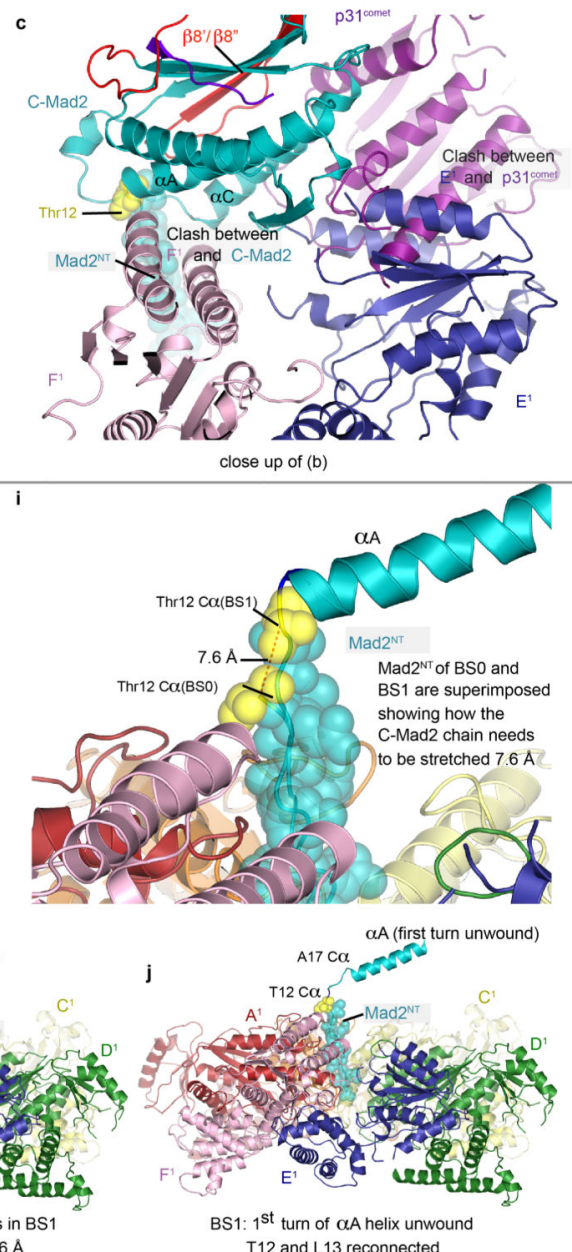

Extended Data Figure 9. Models of the TRIP13:p31 ${ }^{\text {comet }}$ :C-Mad2 complexes in basal state 0 and basal state 1 (pre and post the first catalytic cycle).

a, Basal state 0 (similar to Fig. 4a). Superscripts on TRIP13 subunit labels denote basal state. b, Showing the conformational change of the TRIP13 hexamer post one catalytic cycle

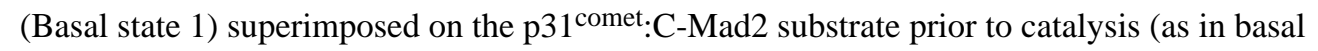
state 0 ). The upward movement of the $\mathrm{E}$ and $\mathrm{F}$ subunits clashes with p31 ${ }^{\text {comet }}$ and C-Mad2. Pore loop residues of E and F subunits shift by $30 \AA$ and $13 \AA$, respectively. c, close up view of (b) showing the clash between the $\mathrm{F}^{1}$ and $\mathrm{E}^{1}$ subunits of TRIP13 in basal state 1 with CMad2 and p31 ${ }^{\text {comet }}$, respectively (as in basal state 0). d, Basal state 1 with TRIP13 and

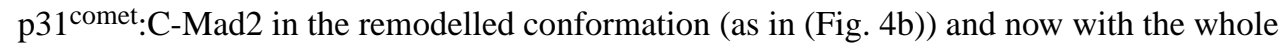
of the p31 ${ }^{\text {comet}}$ :C-Mad2:Cdc20 substrate repositioned onto the new $\mathrm{C}^{1}$ and $\mathrm{D}^{1}$ interface and the aA helix unwound by one turn. e, as in (d) but rotated $60^{\circ}$ to show the $\mathrm{p} 31^{\text {comet: }} \mathrm{C}$ -

Mad2:Cdc20 substrate in the same view as in (a). f-j: Panels showing TRIP13 and only C$\operatorname{Mad} 2^{\mathrm{NT}}$ and connection to the C-Mad2 aA helix and proposed unwinding of the C-Mad2 aA helix. f, BS0 with view as in (e). g, TRIP13 adopts the BS1 conformation as in (e) with 
the whole p31 ${ }^{\text {comet:}} \mathrm{C}-\mathrm{Mad} 2$ substrate repositioned onto the $\mathrm{C}^{1}-\mathrm{D}^{1}$ interface. Note that the aA helix of C-Mad2 has not been unwound and C-Mad2 $2^{\mathrm{NT}}$ (residues 2-12) is shifted along with the globular domain of C-Mad2 $\left(\mathrm{C}-\mathrm{Mad}_{2}{ }^{\mathrm{GD}}\right)$. $\mathbf{h}, \mathrm{BS} 1$ with C-Mad2 ${ }^{\mathrm{NT}}$ in the BS0 position (i.e. unshifted relative to BS0) and $\mathrm{aA}$ of C-Mad2 ${ }^{\mathrm{GD}}$ as p31 ${ }^{\text {comet: }} \mathrm{C}-\mathrm{Mad} 2$ is repositioned on the $\mathrm{C}^{1}-\mathrm{D}^{1}$ interface (but the aA helix is not unwound). This shows the $11 \AA$ break between the Ca-atoms of Thr12 of C-Mad2 ${ }^{\mathrm{NT}}$ and Leu13 of C-Mad2 ${ }^{\mathrm{GD}}$. C-Mad2 ${ }^{\mathrm{NT}}$ is shifted down the TRIP13 pore two residues relative to $(\mathrm{g})$. $\mathbf{i}$, Close up view of the C$\operatorname{Mad} 2^{\mathrm{NT}}$ and connection to the aA helix. The view is the same as in (h) and it superimposes the position of C-Mad2 ${ }^{\mathrm{NT}}$ as in the BS0 and BS1 states showing a 7.6 $\AA$ distance between the $\mathrm{Ca}$-atoms of Thr12 of C-Mad ${ }^{\mathrm{NT}}$ in the two states. This indicates the extent of required unwinding of the aA helix. $\mathbf{j}$, The first turn of the aA helix unwinds to reconnect Thr12 of C-Mad2 ${ }^{\mathrm{NT}}$ with Leu13 of the C-Mad2 a A helix. Model building was guided by insights from cryo-EM structures of the AAA+ ATPases VAT 45, Vps4 46 and Hsp104 47 that indicate a processive hand-over-hand mechanism in which the AAA+ motor translocates along the axis of the substrate. ATP hydrolysis propagates a sequential conformational change within the hexameric ring that converts the conformation of each subunit to that of its clockwise neighbour (viewed from $\mathrm{p} 31^{\text {comet }}$ ).

Extended Data Table 1

EM data collection, processing statistics and structure refinement statistics

\begin{tabular}{|c|c|}
\hline & $\begin{array}{l}\text { TRIP } 13 \text { in complex with ATP-gamma-S, p31comet, C-Mad2 and Cdc20 } \\
\text { (EMDB-4166) } \\
\text { (PDB 6F0X) }\end{array}$ \\
\hline \multicolumn{2}{|l|}{ Data collection and processing } \\
\hline Magnification & 81,000 \\
\hline Voltage (kV) & 300 \\
\hline Electron exposure $\left(\mathrm{e}-/ \AA^{2}\right)$ & 40 \\
\hline Defocus range $(\mu \mathrm{m})$ & $2.0-3.6$ \\
\hline Pixel size $(\AA)$ & 1.43 \\
\hline Symmetry imposed & $\mathrm{CI}$ \\
\hline Initial particle images (no.) & 440,853 \\
\hline Final particle images (no.) & 354,157 \\
\hline Map resolution $(\AA)$ & 4.6 \\
\hline FSC threshold & 0.143 \\
\hline Map resolution range $(\AA)$ & $4.2-5.5$ \\
\hline \multicolumn{2}{|l|}{ Refinement } \\
\hline Initial model used (PDB code) & $5 \mathrm{vqa}, 2 \mathrm{qyf}$ \\
\hline Model resolution $(\AA)$ & 4.2 \\
\hline FSC threshold & - \\
\hline Model resolution range $(\AA)$ & - \\
\hline Map sharpening $B$ factor $\left(\AA^{2}\right)$ & -342.45 \\
\hline \multicolumn{2}{|l|}{ Model composition } \\
\hline Non-hydrogen atoms & 20491 \\
\hline
\end{tabular}




\begin{tabular}{ll}
\hline & $\begin{array}{l}\text { TRIP 13 in complex with ATP-gamma-S, p31comet, C-Mad2 and Cdc20 } \\
\text { (EMDB-4166) } \\
\text { (PDB 6F0X) }\end{array}$ \\
\hline Protein residues & 2541 \\
Ligands & 5 \\
B factors $\left(\AA^{2}\right)$ & - \\
Protein & - \\
Ligand & \\
R.m.s. deviations & 0.01 \\
Bond lengths $(\AA)$ & 1.13 \\
Bond angles $\left({ }^{\circ}\right)$ & \\
Validation & 2.62 \\
MolProbity score & 27.73 \\
Clashscore & 0 \\
Poor rotamers $(\%)$ & \\
Ramachandran plot & 79.26 \\
Favored $(\%)$ & 20.19 \\
Allowed $(\%)$ & 0.55 \\
Disallowed $(\%)$ & \\
\hline
\end{tabular}

\section{Supplementary Material}

Refer to Web version on PubMed Central for supplementary material.

\section{Acknowledgments}

This work was funded by MRC and CR-UK grants to D.B. C.A. is an EMBO Advanced Fellow. We are grateful to A. Boland for comments on the manuscript; S. Chen, C. Savva and G. McMullan for help with the EM data collection; J. Grimmett and T. Darling for computing.

\section{References}

1. Musacchio A. The Molecular Biology of Spindle Assembly Checkpoint Signaling Dynamics. Current biology : CB. 2015; 25:R1002-1018. [PubMed: 26485365]

2. Alfieri C, Zhang S, Barford D. Visualizing the complex functions and mechanisms of the anaphase promoting complex/cyclosome (APC/C). Open biology. 2017; 7

3. Kulukian A, Han JS, Cleveland DW. Unattached kinetochores catalyze production of an anaphase inhibitor that requires a Mad2 template to prime Cdc20 for BubR1 binding. Developmental cell. 2009; 16:105-117. [PubMed: 19154722]

4. Faesen AC, et al. Basis of catalytic assembly of the mitotic checkpoint complex. Nature. 2017; 542:498-502. [PubMed: 28102834]

5. Ji Z, Gao H, Jia L, Li B, Yu H. A sequential multi-target Mps1 phosphorylation cascade promotes spindle checkpoint signaling. eLife. 2017; 6doi: 10.7554/eLife.22513

6. Habu T, Kim SH, Weinstein J, Matsumoto T. Identification of a MAD2-binding protein, CMT2, and its role in mitosis. The EMBO journal. 2002; 21:6419-6428. [PubMed: 12456649]

7. Eytan E, et al. Disassembly of mitotic checkpoint complexes by the joint action of the AAA-ATPase TRIP13 and p31(comet). Proceedings of the National Academy of Sciences of the United States of America. 2014; 111:12019-12024. [PubMed: 25092294] 
8. Wang K, et al. Thyroid hormone receptor interacting protein 13 (TRIP13) AAA-ATPase is a novel mitotic checkpoint-silencing protein. The Journal of biological chemistry. 2014; 289:23928-23937. [PubMed: 25012665]

9. Teichner A, et al. p31comet Promotes disassembly of the mitotic checkpoint complex in an ATPdependent process. Proceedings of the National Academy of Sciences of the United States of America. 2011; 108:3187-3192. [PubMed: 21300909]

10. Westhorpe FG, Tighe A, Lara-Gonzalez P, Taylor SS. p31 comet-mediated extraction of Mad2 from the MCC promotes efficient mitotic exit. Journal of cell science. 2011; 124:3905-3916. [PubMed: 22100920]

11. Reddy SK, Rape M, Margansky WA, Kirschner MW. Ubiquitination by the anaphase-promoting complex drives spindle checkpoint inactivation. Nature. 2007; 446:921-925. [PubMed: 17443186]

12. Foster SA, Morgan DO. The APC/C subunit Mnd2/Apc15 promotes Cdc20 autoubiquitination and spindle assembly checkpoint inactivation. Molecular cell. 2012; 47:921-932. [PubMed: 22940250]

13. Mansfeld J, Collin P, Collins MO, Choudhary JS, Pines J. APC15 drives the turnover of MCCCDC20 to make the spindle assembly checkpoint responsive to kinetochore attachment. Nature cell biology. 2011; 13:1234-1243. [PubMed: 21926987]

14. Uzunova K, et al. APC15 mediates CDC20 autoubiquitylation by APC/C(MCC) and disassembly of the mitotic checkpoint complex. Nature structural \& molecular biology. 2012; 19:1116-1123.

15. Jia L, et al. Defining pathways of spindle checkpoint silencing: functional redundancy between Cdc20 ubiquitination and p31(comet). Molecular biology of the cell. 2011; 22:4227-4235. [PubMed: 21937719]

16. Eytan E, Sitry-Shevah D, Teichner A, Hershko A. Roles of different pools of the mitotic checkpoint complex and the mechanisms of their disassembly. Proceedings of the National Academy of Sciences of the United States of America. 2013; 110:10568-10573. [PubMed: 23754430]

17. Ye QZ, et al. TRIP13 is a protein-remodeling AAA plus ATPase that catalyzes MAD2 conformation switching. eLife. 2015; 4

18. Ye Q, et al. The AAA+ ATPase TRIP13 remodels HORMA domains through N-terminal engagement and unfolding. The EMBO journal. 2017; 36:2419-2434. [PubMed: 28659378]

19. Yang M, et al. p31comet blocks Mad2 activation through structural mimicry. Cell. 2007; 131:744755. [PubMed: 18022368]

20. Ma HT, Poon RY. TRIP13 Regulates Both the Activation and Inactivation of the Spindle-Assembly Checkpoint. Cell reports. 2016; 14:1086-1099. [PubMed: 26832417]

21. Xia G, et al. Conformation-specific binding of p31(comet) antagonizes the function of Mad2 in the spindle checkpoint. The EMBO journal. 2004; 23:3133-3143. [PubMed: 15257285]

22. Mapelli M, et al. Determinants of conformational dimerization of Mad2 and its inhibition by p31 comet. The EMBO journal. 2006; 25:1273-1284. [PubMed: 16525508]

23. Mapelli M, Massimiliano L, Santaguida S, Musacchio A. The Mad2 conformational dimer: structure and implications for the spindle assembly checkpoint. Cell. 2007; 131:730-743. [PubMed: 18022367]

24. Lyubimov AY, Strycharska M, Berger JM. The nuts and bolts of ring-translocase structure and mechanism. Current opinion in structural biology. 2011; 21:240-248. [PubMed: 21282052]

25. Yang M, et al. Insights into mad 2 regulation in the spindle checkpoint revealed by the crystal structure of the symmetric mad2 dimer. PLoS biology. 2008; 6:e50. [PubMed: 18318601]

26. Luo X, et al. The Mad2 spindle checkpoint protein has two distinct natively folded states. Nature structural \& molecular biology. 2004; 11:338-345.

27. Hara M, Ozkan E, Sun H, Yu H, Luo X. Structure of an intermediate conformer of the spindle checkpoint protein Mad2. Proceedings of the National Academy of Sciences of the United States of America. 2015; 112:11252-11257. [PubMed: 26305957]

28. Alfieri C, et al. Molecular basis of APC/C regulation by the spindle assembly checkpoint. Nature. 2016; 536:431-436. [PubMed: 27509861]

29. Zheng SQ, et al. MotionCor2: anisotropic correction of beam-induced motion for improved cryoelectron microscopy. Nature methods. 2017; 14:331-332. [PubMed: 28250466] 
30. Zhang K. Gctf: Real-time CTF determination and correction. Journal of structural biology. 2016; 193:1-12. 3. [PubMed: 26592709]

31. Fernandez-Leiro R, Scheres SHW. A pipeline approach to single-particle processing in RELION. Acta crystallographica. Section D, Structural biology. 2017; 73:496-502. [PubMed: 28580911]

32. Afanasyev P, et al. Single-particle cryo-EM using alignment by classification $(A B C)$ : the structure of Lumbricus terrestris haemoglobin. IUCrJ. 2017; 4:678-694.

33. Chen $\mathrm{S}$, et al. High-resolution noise substitution to measure overfitting and validate resolution in 3D structure determination by single particle electron cryomicroscopy. Ultramicroscopy. 2013; 135:24-35. [PubMed: 23872039]

34. Yang Z, et al. UCSF Chimera, MODELLER, and IMP: an integrated modeling system. Journal of structural biology. 2012; 179:269-278. [PubMed: 21963794]

35. Emsley P, Cowtan K. Coot: model-building tools for molecular graphics. Acta crystallographica. Section D, Biological crystallography. 2004; 60:2126-2132. [PubMed: 15572765]

36. Zhang F, et al. Structural insights into the regulatory particle of the proteasome from Methanocaldococcus jannaschii. Molecular cell. 2009; 34:473-484. [PubMed: 19481527]

37. Adams PD, et al. PHENIX: a comprehensive Python-based system for macromolecular structure solution. Acta crystallographica. Section D, Biological crystallography. 2010; 66:213-221. [PubMed: 20124702]

38. Landau M, et al. ConSurf 2005: the projection of evolutionary conservation scores of residues on protein structures. Nucleic acids research. 2005; 33:W299-302. [PubMed: 15980475]

39. Krissinel E, Henrick K. Inference of macromolecular assemblies from crystalline state. Journal of molecular biology. 2007; 372:774-797. [PubMed: 17681537]

40. Waterhouse AM, Procter JB, Martin DMA, Clamp M, Barton GJ. Jalview Version 2-a multiple sequence alignment editor and analysis workbench. Bioinformatics. 2009; 25:1189-1191. [PubMed: 19151095]

41. Kucukelbir A, Sigworth FJ, Tagare HD. Quantifying the local resolution of cryo-EM density maps. Nature methods. 2014; 11:63-65. [PubMed: 24213166]

42. Chao WC, Kulkarni K, Zhang Z, Kong EH, Barford D. Structure of the mitotic checkpoint complex. Nature. 2012; 484:208-213. [PubMed: 22437499]

43. Luo X, Tang Z, Rizo J, Yu H. The Mad2 spindle checkpoint protein undergoes similar major conformational changes upon binding to either Mad1 or Cdc20. Molecular cell. 2002; 9:59-71. [PubMed: 11804586]

44. Sironi L, et al. Crystal structure of the tetrameric Mad1-Mad2 core complex: implications of a 'safety belt' binding mechanism for the spindle checkpoint. The EMBO journal. 2002; 21:24962506. [PubMed: 12006501]

45. Ripstein ZA, Huang R, Augustyniak R, Kay LE, Rubinstein JL. Structure of a AAA+ unfoldase in the process of unfolding substrate. eLife. 2017; 6

46. Monroe N, Han H, Shen PS, Sundquist WI, Hill CP. Structural basis of protein translocation by the Vps4-Vta1 AAA ATPase. eLife. 2017; 6

47. Gates SN, et al. Ratchet-like polypeptide translocation mechanism of the AAA+ disaggregase Hsp104. Science. 2017; 357:273-279. [PubMed: 28619716] 

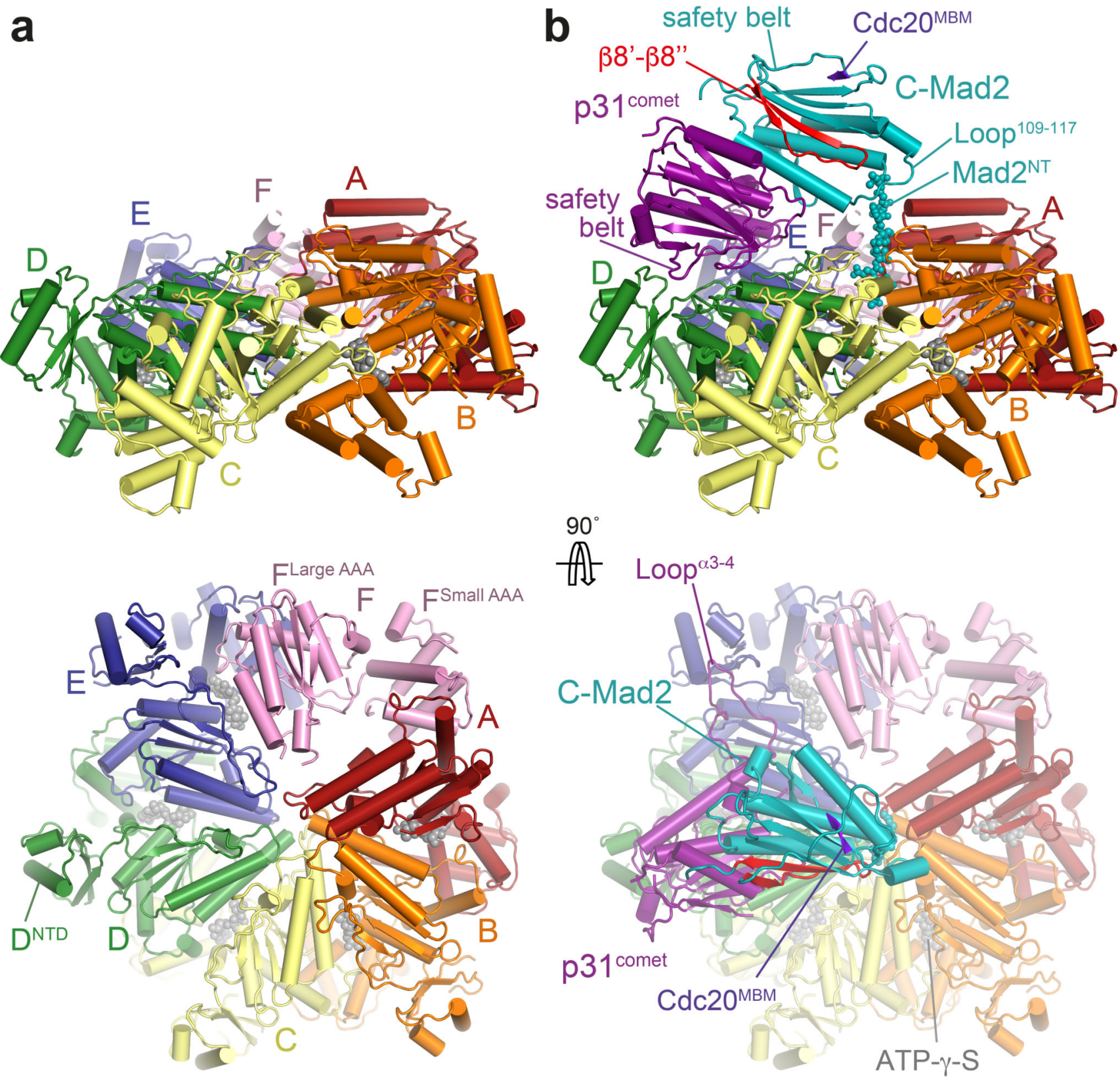

Figure 1. Overall structures of the apo and TRIP13:p31-substrate complexes.

Side and top views of the cryo-EM structure model of TRIP13 in the apo state (a) and in complex with p31 ${ }^{\text {comet}}$ :C-Mad2 (class 2: basal state) (b). Relevant regions of TRIP13, p3 $1^{\text {comet }}$ and C-Mad2 are indicated. 


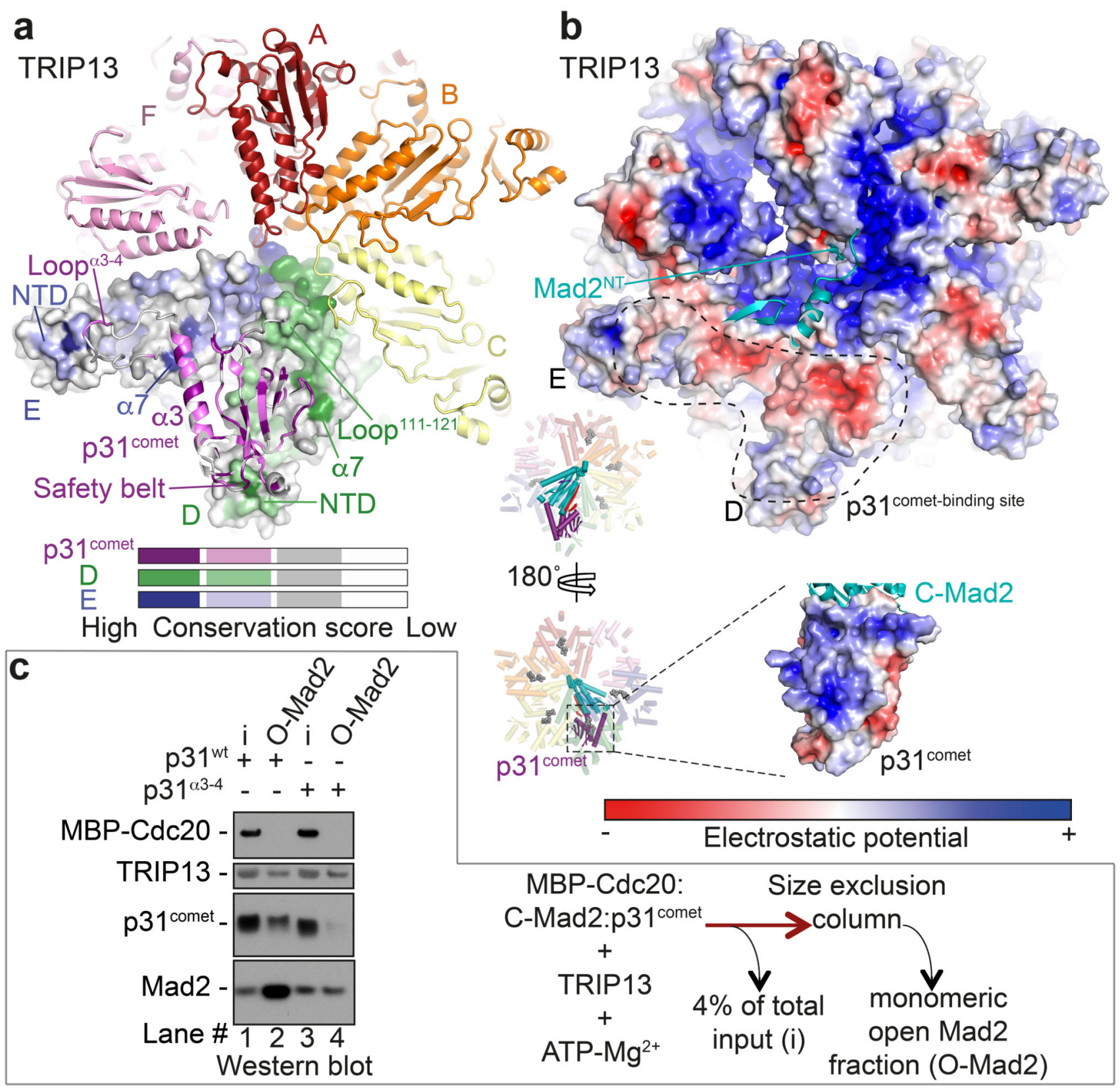

Figure 2. Interaction between p11 $^{\text {comet }}$ and TRIP13.

a, Surface conservation is displayed for TRIP13 monomers D and E, conservation score colour code is indicated below. A portion of $\mathrm{p} 31^{\text {comet }}$ is show in cartoon representation. NTD: N-terminal domain. b, Electrostatic surface representation for the TRIP13 - p31 $1^{\text {comet }}$ interacting surfaces (surface potential at $\pm 5 \mathrm{kT} / \mathrm{e}$ ), colour code is displayed below. Top: TRIP13 electrostatic surface with a portion of C-Mad2 show in cartoon representation. Monomers $\mathrm{D}$ and $\mathrm{E}$ form a continuous acidic patch that is unique to this monomer pairing due to the conformation of loop 111-121 of monomer E. Bottom: p31 ${ }^{\text {comet }}$ electrostatic surface as viewed by TRIP13. Cartoons to the left provides an overall perspective. The 
surface area buried at the $\mathrm{p} 31^{\text {comet }}$-TRIP13 interface is $2,392 \AA^{2}$. c, Deletion of conserved basic residues of the p31 $1^{\text {comet }}$ a 3-4 loop (Extended Data Fig. 7b) severely disrupts TRIP13p31 $1^{\text {comet }}$-catalysed Mad2 remodelling. Right: experimental design for Cdc20:C-Mad2 complex disassembly reaction. Left: Western blot showing input (i) and size exclusion fractions corresponding to monomeric O-Mad2 (lanes 2 and 4) and Extended Data Fig. 2c. Experiment in $\mathbf{c}$ was in triplicate with similar results. For gel source data see Supplementary Fig. 1. 


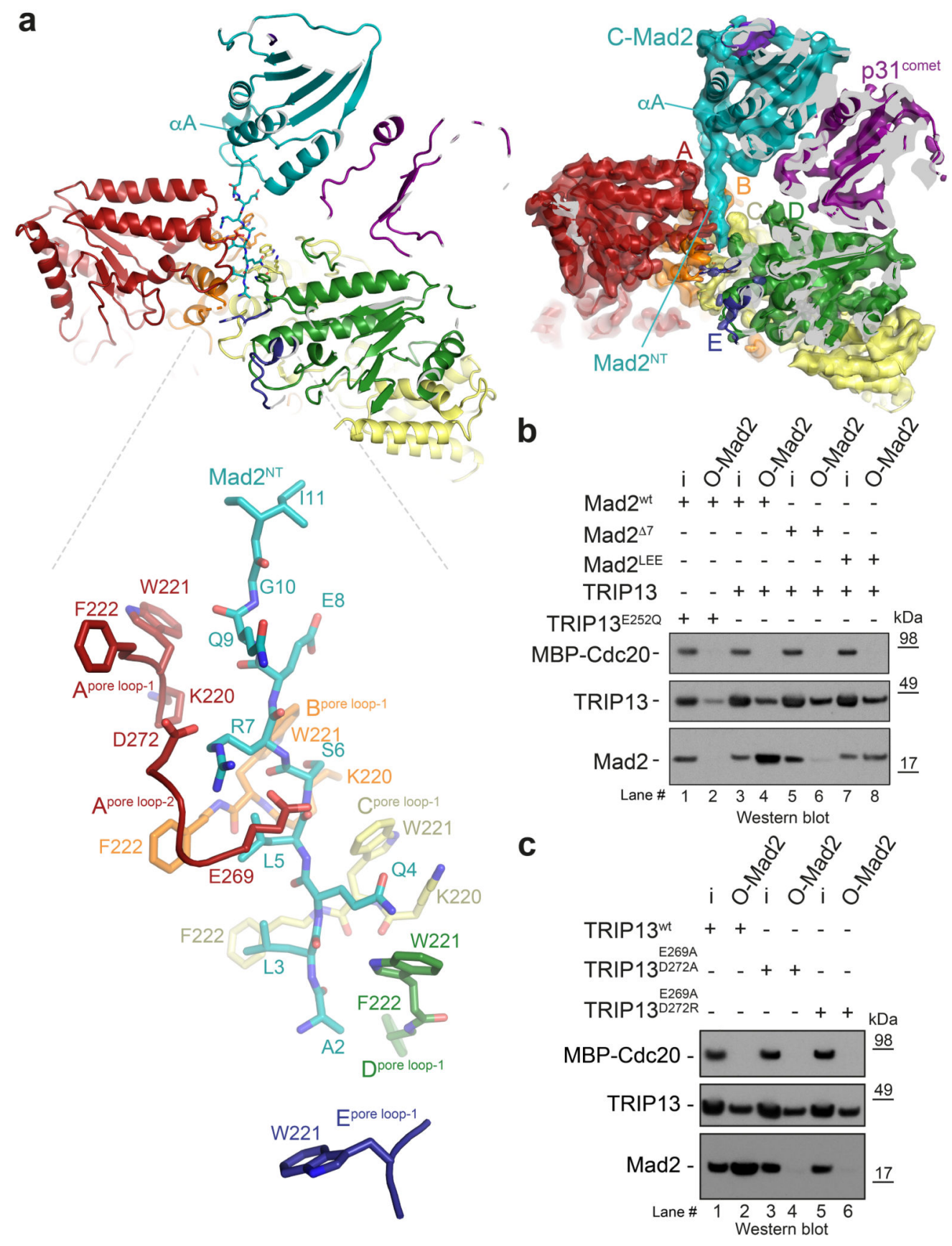

Figure 3. Interaction between C-Mad2 ${ }^{\mathrm{NT}}$ and the TRIP13 pore loops.

a, Top: Overview of the TRIP13:p31-substrate complex showing Mad2 ${ }^{\mathrm{NT}}$ inserted into the TRIP13 pore (left) with corresponding EM density map on right. Bottom: Detailed representation of $\mathrm{Mad}_{2}{ }^{\mathrm{NT}}$ residues interacting with TRIP13 pore loop residues. b, Western blot showing input (i) and O-Mad2 fractions of the Cdc20:C-Mad2 complex disassembly by TRIP13 ${ }^{\mathrm{E} 253 \mathrm{Q}}$ mutant (lanes 1-2) and TRIP13 ${ }^{\mathrm{wt}}$ (lanes 3-8). Mad2 levels and loading controls for TRIP13 and Cdc20 are detected with their respective antibodies. Mutants of $\mathrm{Mad}^{\mathrm{NT}}$ (lanes 5-8) affect the levels of O-Mad2 released from the Cdc20:C-Mad2 complex. 
c, Mutation of D269 and E272 of pore loop-2 of TRIP13 ablates TRIP13-p31 ${ }^{\text {comet-catalysed }}$ Mad2 remodelling (and Extended Data Fig. 2c). Experiments in b, $\mathbf{c}$ were in triplicate with similar results. 

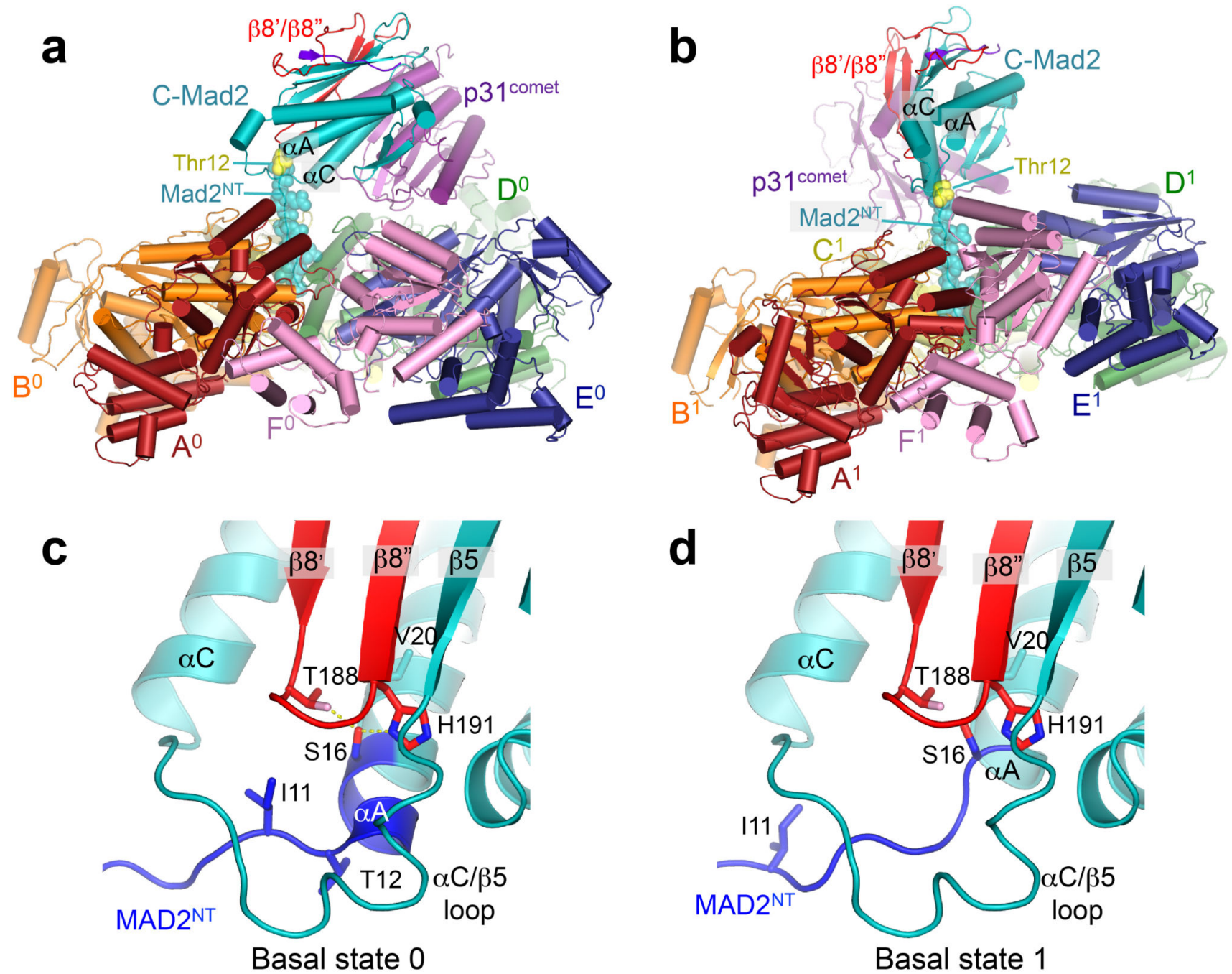

Figure 4. Sequential catalytic cycles of TRIP13 remodel Mad2.

a, b, Overall views of TRIP13:p31-substrate complex: (a) before catalysis (pre-catalytic: basal state 0) and (b) after the first catalytic cycle (basal state 1). TRIP13 subunit superscripts denote the catalytic cycle. Thr12 of Mad2 ${ }^{\mathrm{NT}}$ is coloured yellow indicating the boundary with aA in basal state 0 . After the first catalytic cycle one turn of the aA helix unwinds (compare (c) and (d)). This disrupts contacts between the N-terminus of the aA helix and the $\beta 8$ ' $-\beta 8$ "' hairpin. The N-terminal region of C-Mad 2 that differs between OMad2 and C-Mad2 is in blue (Extended Data Fig. 6b,c). Further structural details are in Extended Data Fig. 9. 


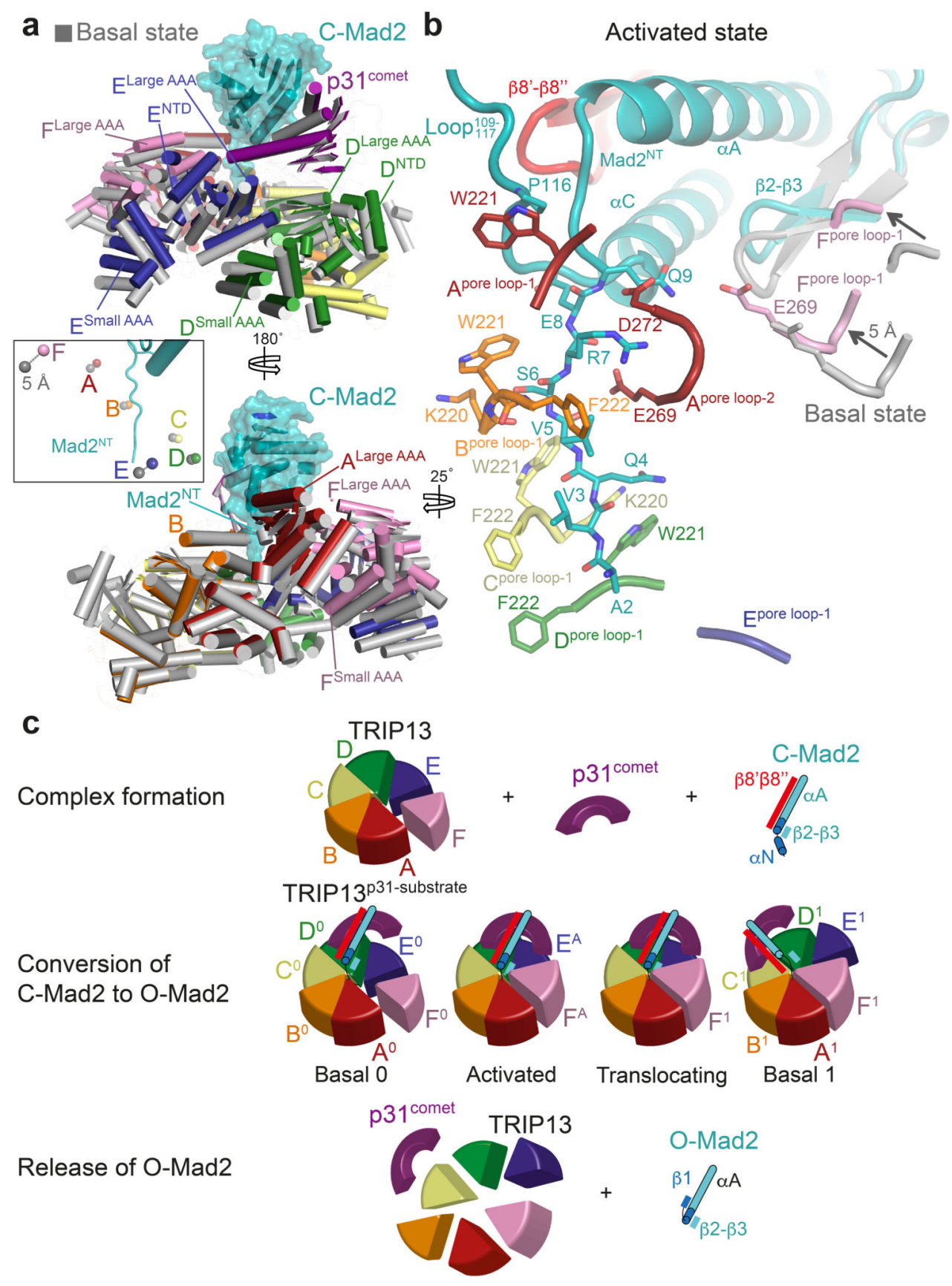

Figure 5. Differences between basal and activated states of the TRIP13-p31-substrate.

a, Two views comparing the basal state (grey) and activated state (colour code as in Fig. 1) of the TRIP13:p31-substrate (superimposition on C-Mad2). Left insert panel illustrates displacement of reference pore loop residues between basal and active states. The displacement grows from the $\mathrm{p} 31^{\text {comet }}$-contacting monomer $\mathrm{D}$ to the F monomer, with $\mathrm{F}$ featuring the largest displacement of $5 \AA$. b, The conformational changes of monomer $\mathrm{F}$ pore loops and the C-Mad2 $\beta 2-\beta 3$ hairpin in the active state are shown relative to their positions in the basal state (grey). c, Schematic of the proposed mechanism of TRIP13- 
p31 $1^{\text {comet }}$-catalysed remodelling of O-Mad2 (and Supplementary Video 2). The remodelled structural elements of $\mathrm{Mad} 2$ are indicated. $\mathrm{E}^{\mathrm{A}}$ and $\mathrm{F}^{\mathrm{A}}$ : activated conformations of monomers $\mathrm{E}$ and $\mathrm{F}$, respectively. The O-Mad2 products dissociate from $\mathrm{p} 31^{\text {comet. }}$ 